\title{
Correlating electrochemical impedance with hierarchical structure for porous carbon-based supercapacitors using a truncated transmission line model
}

\author{
Dina Ibrahim Abouelamaiem ${ }^{\mathrm{a}}$, Guanjie $\mathrm{He}^{\mathrm{b}}$, Tobias P. Neville ${ }^{\mathrm{a}}$, Drasti Patel ${ }^{\mathrm{a}}$, Shan Jic, \\ Rongfang Wang ${ }^{\mathrm{d}}$, Ivan P. Parkin ${ }^{\mathrm{b}}$, Ana Belen Jorge ${ }^{\mathrm{e}}$, Maria-Magdalena Titirici ${ }^{\mathrm{e}}$, Paul R. \\ Shearing $^{\mathrm{a}}$, Daniel J. L. Brett ${ }^{* a}$ \\ ${ }^{a}$ Electrochemical Innovation Lab, Department of Chemical Engineering, University College London, \\ London, WC1E 7JE, UK \\ ${ }^{b}$ Christopher Ingold Laboratory, Department of Chemistry, University College London, London, \\ WCIH OAJ, UK \\ ${ }^{c}$ College of Biological, Chemical Sciences and Engineering, Jiaxing University, 314001,China \\ ${ }^{d}$ Qingdao University of Science and Technology, Institute of Chemical Engineering, Qingdao,266000, \\ China \\ ${ }^{e}$ Materials Research Institute, School of Engineering and Materials Science, Queen Mary University \\ of London, London, E1 4NS, UK
}

\section{*Corresponding Author, e-mail: $\underline{\text { d.brett@ucl.ac.uk }}$}

\section{Keywords}

Electrochemical impedance spectroscopy; Equivalent circuit; Pore size distribution; Relaxation time; Transmission line model

\begin{abstract}
This work considers the relationship between the morphology of porous carbon materials used for supercapacitors and the electrochemical impedance spectroscopy (EIS) response. EIS is a powerful tool that can be used to study the porous 3-dimensional electrode behavior in different electrochemical systems. Porous carbons prepared by treatment of cellulose with different compositions of potassium hydroxide $(\mathrm{KOH})$ were used as model systems to investigate the form vs. electrochemical function relationship. A simple equivalent circuit that represents the electrochemical impedance behavior over a wide range of frequencies was designed. The associated impedances with the bulk electrolyte, Faradaic electrode processes and different pore size ranges were investigated using a truncated version of the standard transmission line model. The analysis considers the requirements of porous materials as
\end{abstract}


electrodes in supercapacitor applications, reasons for their non-ideal performance and the concept of 'best capacitance' behavior in different frequency ranges.

\section{Introduction}

Carbon is extensively used as an electrode material or catalyst support in electrochemical energy conversion and storage devices due to its versatile properties. This includes its existence in various microstructures, different degrees of graphitization and ease of manipulation [1-3]. More specifically, activated carbons have been the primary candidate for electrode materials in electrical double-layer capacitors (EDLCs). Supercapacitors have been intensively investigated as they bridge the gap between conventional capacitors and batteries, with the ability to provide instant energy for short periods with high specific power rates [35]. Supercapacitors can be classified into two categories: i) EDLCs, in which capacitance arises from the accumulation of electrostatic charge at the electrode/electrolyte interphase; and ii) pseudo-capacitors, whereby reversible Faradaic processes, governed by surface electro-active species, provide the energy storage mechanism [6, 7].

Large specific surface area, a hierarchically structured pore size distribution (PSD) and a high proportion of micropores, are the primary factors that dictate the high performance of doublelayer capacitors [8]. Recent studies have recorded high specific capacitance values with excellent cyclability and stability, owing to the use of innovative materials with optimized surface properties and porous texture [9-11]. However, there has been little work reported on the underlying factors that determine the relationship between structural form and electrochemical performance [12-14].

Electrochemical impedance spectroscopy (EIS) is a powerful diagnostic tool used to deconvolute the various frequency dependant impedances in electrochemical systems. 
However, there is much more scope for applying EIS to supercapacitors to understand the frequency response of porous carbon materials and their corresponding capacitive behavior. The focus of this work is divided into two main categories: i) developing a simple equivalent circuit that describes the performance of porous electrodes in supercapacitors using $\mathrm{KOH}$ activated carbon materials as model systems, and ii) assessing the role of the morphology/pore structure in determining non-ideal performances and enhancing the electrochemical performance of double-layer capacitors. Different PSDs and micropore volume fractions are investigated to show how this affects the electrochemical impedance behavior and the relaxation times in different porous carbon networks.

Supercapacitor electrode materials composed of porous carbon materials, whether in an activated carbon matrix, carbon aerogel or carbon nanotube forms, exhibit various internal resistances that contribute to the total resistance of the equivalent resistance circuit [15]. The electronic resistance element is comprised of the electrode material resistance (interparticle and intraparticle resistances), electrode/current collector contact resistance and the electrode/separator resistance. As for the ionic resistance, this comprises the bulk electrolyte resistance, the electrode/electrolyte 'external' surface resistance and the 'internal' electrolyte resistance within the different sized pores [16-18]. The charge transfer resistance governs the Faradaic processes, commonly induced in carbon electrodes due to i) redox reactions with surface functionalities or pseudocapacitive elements, and/or ii) overcharge/overdischarge leakage resistance. Each of the individual resistances contributes to the EIS profile and can be modelled using an equivalent circuit.

Ion transport in three-dimensional porous electrodes must be considered differently to 'twodimensional' electrochemical processes on the 'flat' surface of planar electrodes, where semiinfinite diffusion can generally be assumed [19]. Hence, 'ideal' finite-length Warburg 
impedance elements cannot be applied to the porous electrodes used in supercapacitors in the same way as planar electrodes. The phase angle between the applied potential at the pore orifice and the charging current at low frequency has a similar behavior to that of the Warburg diffusion impedance of a planar electrode in solutions of low concentrations. However, the distribution of resistances originating from various pore channel sizes can be used to model the non-uniform current distribution, and to classify the dispersion of capacitance in the porous matrix over a wide range of frequencies. A 'universal' equivalent circuit based on a transmission line model (TLM), has been suggested to represent the electrochemical impedance behavior of porous materials [19]. In this arrangement, a network of parallel resistance $(R)$ and capacitance $(C)$ elements, each with its own time constant, combine to represent the impedance behavior of a porous electrode. Fletcher et. al then developed the universal equivalent circuit through software and hardware realizations of their model in which it was validated via comparison of the developed "Pascal equivalent circuits" and real supercapacitor performances [20].

A limitation of the use of complex equivalent circuits is that plausible fits can be achieved to EIS data using a range of different circuit element values. Such 'degenerate networks' can establish identical impedance values over an entire range of frequencies and therefore identifying the behavior of a specific complex system is not trivial. While the likes of Fletcher has derived transformation formulas for common 'degenerate networks' to solve the ambiguity of different electrical models [21], the more complicated the equivalent circuit, the greater the inherent uncertainty in the determination of fitted parameters. Thus it is normally preferable to apply the simplest equivalent circuit possible to characterize a system.

A truncated version of the universal equivalent circuit was proposed for porous electrodes, wherein non-branching pores are assumed throughout the porous matrix [22]. This approach 
resolves the issue of selecting the "best" fitting circuit, regardless of the number and relevance of the elements in the candidate models considered, where the number $n$ and values of the " $R C$ " vertical ladder is chosen arbitrarily. However, it might not represent all the different PSDs in the porous electrode and therefore not have a direct correspondence with the real physical values of the modelled circuit.

An equivalent circuit for a single pore was first proposed by de Levie, in which an ideal pore has a solution resistance $R$, and a double-layer capacitance $C$ that behaves equivalently to the TLM [23]. Modifications and simplifications of the TLM followed; these methods have used different assumptions based on the precursor materials and corresponding morphological structures $[24,25]$. Table 1 summarizes the suggested equivalent circuit models for EDLCs, along with the assumptions made and elements governing the models. 
Table 1: Summary of equivalent circuits suggested in the literature showing the different model elements and different assumptions made, with resistance $(R)$, capacitance $(C)$, frequency $(f)$, voltage $(V)$, and current $(i)$.

\begin{tabular}{|c|c|c|}
\hline Equivalent series circuit suggested & Elements & Assumptions \\
\hline 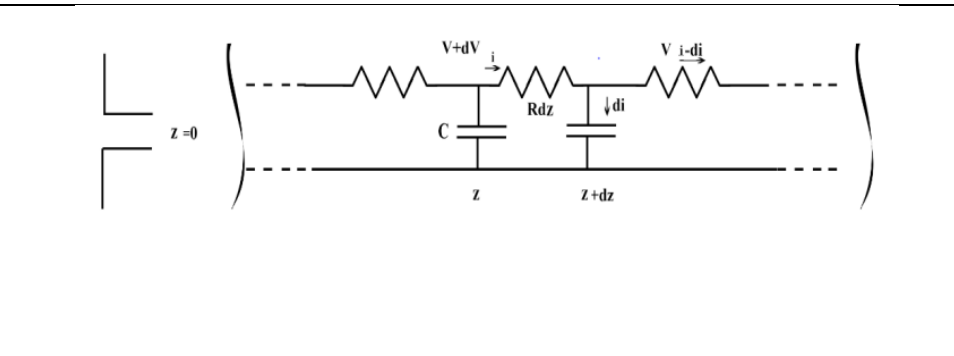 & $\begin{array}{l}d z: \text { section of equivalent circuit of } \\
\text { single pore; } R: \text { solution } R ; C \text { : } \\
\text { double-layer } C \text { inside the pore; } V \text { : } \\
\text { voltage; } i \text { : current. }\end{array}$ & $\begin{array}{l}\text { De-Levie: A model of an element of a single } \\
\text { pore; cylindrical with uniform radius; } R \& C \\
\text { uniformly distributed down the pore, both } \\
\text { expressed per unit length; current conducting } \\
\text { matrix of negligible } R \text {. [23] }\end{array}$ \\
\hline 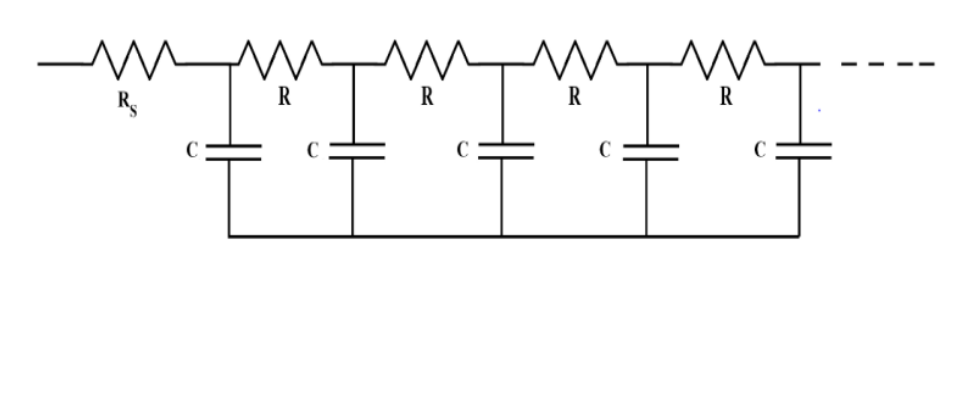 & $\begin{array}{l}R_{s}: \text { electrolyte } R \text { outside pores; } R \text { : } \\
\text { electrolyte } R \text { inside pores; } C \text { : } \\
\text { double-layer. }\end{array}$ & $\begin{array}{l}\text { TLM: } R C \text {-parallel elements with well-chosen } \\
\text { numbers of 'values'; Faradaic reactions are } \\
\text { absent; cylindrically porous carbons; curvature } \\
\text { is neglected; uniform pores; frequency- } \\
\text { response exhibits }-45^{\circ} \text { phase angle; behavior is } \\
\text { ideal capacitor at low } f \text {. [19] }\end{array}$ \\
\hline
\end{tabular}



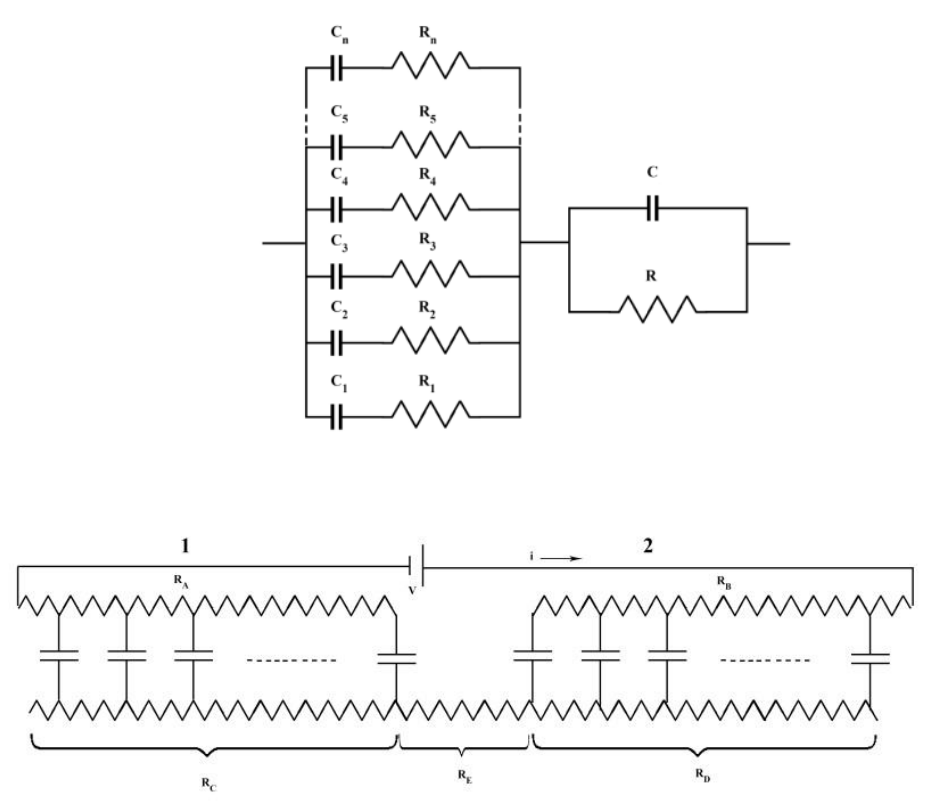

$R_{i}, C_{i}$ : vertical ladder network of Any set of non-uniform pores in parallel; $R C$-series components of electrolyte correspondence is not one-to-one with physical $R$ inside pores and $C$ at patch of pore values; proposed circuit is degenerate with walls; $R \& C$ : bulk solution $R$ and another random set of parallel $R C$-elements; dielectric polarization $C$ of solution. electrolyte $R$ in pores is higher than $R$ of solid carbons; asynchronous charging. [22]

$R_{A}: R$ of electron flow in porous No direct flow of charges from carbon into carbons of electrode $1 ; R_{C}: R$ of ion electrolyte; electrode/electrolyte interface is flow in pore solution in electrode 1; similar to plane-electrodes; electrolyte flows $C$ : double-layer $C ; R_{B} ; R_{D}$ : same as between particles but not through them; $R_{A}$ and $R_{C}$ on electrode $2 ; V$ : voltage micropore surface accessible at any rate; effect of power source; i: current; $R_{E}$ : of concentration on $V$ and charge is neglected; separator $R$. double-layer at local equilibrium; charge transfer ignored; concentration and $V$ of electrolyte are uniform across pore crosssection. [26] 

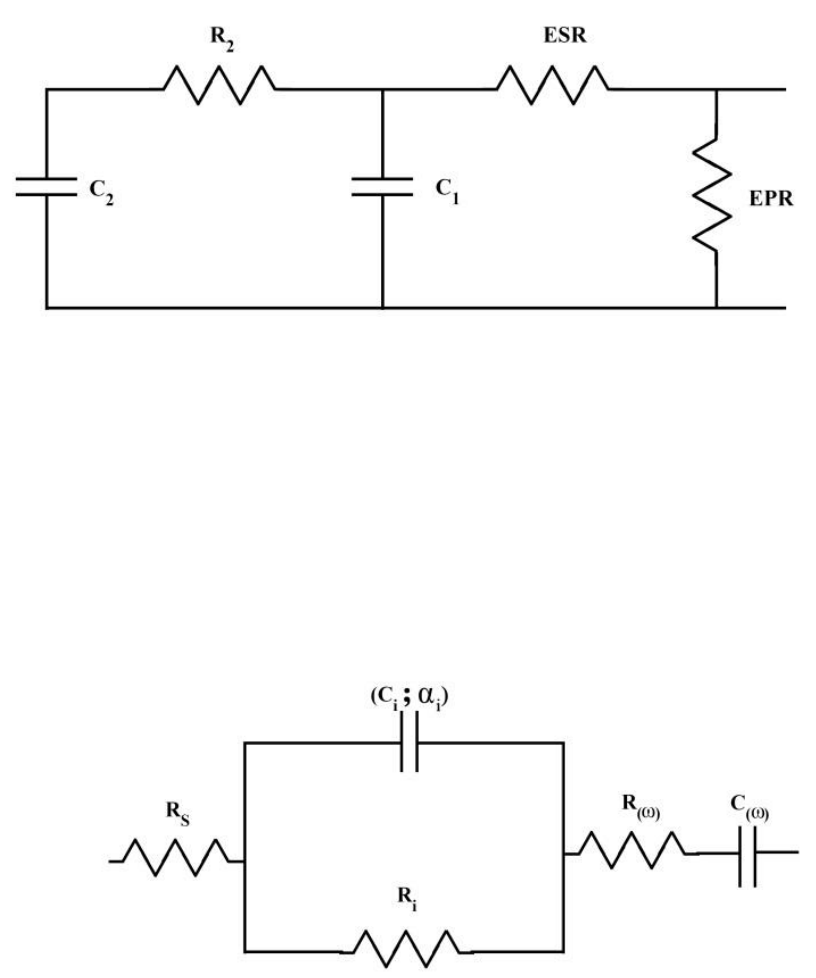

$2 R C$ parallel branches; $C_{l}$ : Intermediate $\tau$ omitted; specific for cases when capacitor; ESR: equivalent series $V$ rebounds due to charge redistribution after resistance (small $\tau$ ); EPR: equivalent discharging fully charged $C$; based on TLM; $\tau$ parallel resistor (high $\tau \neq$ constant) in each branch is average of $\tau$ 's for all pore accounts for long-term self- systems; independent of size of $C ; R C$ values in discharge; $C_{2}$ : capacitor and $R_{2}$ : each branch depend on amount of porous $\left(R_{2}>>E S R\right)$.

materials and physical arrangement in electrode. [27]

$R_{s}:$ high $f$ resistance; $R_{i}$ : active At high $f$ : cell behaves on the loop represented material/current collector interface by parallel $R C$ circuit with $R_{s}$ delaying or $R$; $C_{i}$ : interface $C$ with dispersion blocking capacitive behavior; at low f: cell parameter $\alpha_{i} ; \quad R(\omega)$ : partial $R$ represented by $R(\omega)$ having total $R$; depending on $f ; \quad C(\omega)$ : continuation of de Levie's work. [28]

supercapacitor $C$. 


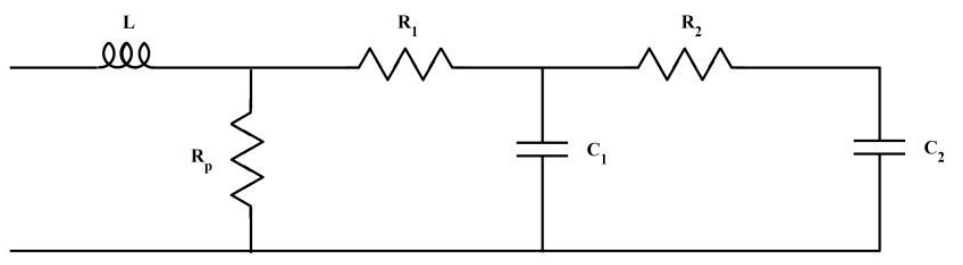

$2 R C$ branches; $C_{1}$ : voltage- Models different charge transfers; $C_{1}$ varies dependent $C$; $R_{l}$ : equivalent series $R$; linearly with $V ; R_{l} C_{l}$ dominates response ( $\tau$ in $C_{2}$ : charge transfer $C ; R_{2}$ : charge $\left.\mathrm{sec}\right) ; R_{1} C_{1}$ determines energy evolution during transfer $R ; R_{p}$ : equivalent parallel $R$. half-cycles in power electronics; $R_{2} C_{2}$ governs slow responses and compliments $R_{l} C_{l}$ in $\tau$ of mins; $R_{2} C_{2}$ governs internal energy distribution at the end of half-cycles; $R_{p}$ only in long-term storage that governs leakage effect \& neglected during fast half-cycles; inductance is neglected; process of each branch observed separately by measuring $V$. [29] 
To the authors' knowledge, EIS using such equivalent circuits has not been used to examine the correlation between morphology and electrochemistry across a range of porous electrode structures. To this end, the effect of different porous networks and PSDs on the capacitive behavior over a wide frequency range has been studied for the first time herein.

A trade-off between minimization of pore resistance and maximizing pore capacitance is discussed based on the use of model systems composed of different relaxation times $(\tau)$. The average pore sizes were identified, categorized as being in the order: i) micropore range $(<2$ $\mathrm{nm})$, ii) mesopore range $(2-50 \mathrm{~nm})$ or iii) macropore range $(>50 \mathrm{~nm})$, and the corresponding relaxation times are all expressed by the equation:

$$
\tau=R C
$$

where $R$ and $C$ describe the resistances and capacitances, respectively, associated with the different aspects of the occuring processes, as will be detailed throughout this work.

A simplified equivalent circuit model is suggested for porous network structures. It has proven to govern the behavior of porous carbons of different morphologies in supercapacitor devices. In this study, cellulose precursors activated with different $\mathrm{KOH}$ loadings were used as the model systems. The various porous structures attained with different $\mathrm{KOH} /$ cellulose ratios were analyzed with different characterization techniques, and their corresponding effect on the impedance spectra is reported. This work can be extended to different porous materials with different pore size distributions and morphologies that can be used in supercapacitors and other electrochemical devices.

\section{Experimental}

\subsection{Synthesis and characterization}


Bio-derived materials have gained increasing interest in the energy conversion and storage devices field due to their abundance, sustainability and reproducibility [30]. Different precursor materials, including coconut shells [31], banana peels [32] and chicken feathers [33] have been employed as electrode materials in EDLCs. These materials have been established as model systems and are easily tuned to achieve porous carbons of high specific surface areas that enhance the electrochemical performance of supercapacitors. Herein, cellulose of softwood pulp origin (Pinus Silvestris and Picea Abies, $\alpha$-cellulose $>85 \%$ ) was activated using different KOH (Fisher Scientific, UK) loadings, as previously reported [12]. Briefly, the cellulose samples (UPM-Kymmene Corporation, Finland) were prepared via blending with water to reach $1 \%$ consistency and $500 \mathrm{~g} \mathrm{~m}^{-2}$. The samples were pressed at a pressure of $5000 \mathrm{lbs}$ and temperature of $120{ }^{\circ} \mathrm{C}$ (Carver Inc., USA), then soaked in $\mathrm{KOH}$ solutions of different concentrations followed by carbonization at a heating ramp of $20{ }^{\circ} \mathrm{C}$ $\min ^{-1}$ up to $850{ }^{\circ} \mathrm{C}$ for $1 \mathrm{~h}$ in $1 \mathrm{~L} \mathrm{~min}^{-1}$ nitrogen flow. The carbonized samples were washed several times to remove all carbonates produced and vacuum-dried at $110{ }^{\circ} \mathrm{C}$ overnight, prior to electrode paste preparation. All $\mathrm{KOH} /$ cellulose samples are denoted as $\mathrm{KOH}-n$ where $n$ represents the $\mathrm{KOH} /$ cellulose mass ratio.

The surface morphologies of all samples with and without chemical activation $(\mathrm{KOH}-0)$ were characterized with scanning electron microscopy (SEM) at $10 \mathrm{kV}$ in secondary electron imaging mode (EVO MA10, ZEISS, Germany) and transmission electron microscopy (TEM) at $200 \mathrm{kV}$ accelerating voltage (JEOL, JEM-2100, UK).

X-ray photoelectron spectra were measured using K-ALPHA Surface Analysis spectrometer (Thermo Scientific) with monochromatic $\mathrm{Al} \mathrm{K} \alpha$ radiation of $1486.6 \mathrm{eV}$ as an excitation source to detect the oxygen and carbon content. The curve fitting and background correction were done using Casa XPS software. Nitrogen adsorption-desorption isotherms (3Flex Surface and Catalyst Characterization System, Micromeritics, USA) were carried out to 
determine the PSD in the microporous and mesoporous size ranges as well the total pore volume in these pore size ranges. The volume was evaluated at a relative pressure $(\mathrm{P} / \mathrm{Po})$ of 0.99 , and the microporous volumes were calculated by non-local density functional theory (NLDFT). Specific surface areas (SSA) were obtained by applying the Brunauer-EmmetTeller (BET) method, calculated at relative pressure range between 0.001 and 0.2 . The samples were characterized by $\mathrm{CO}_{2}$ isotherms at $273 \mathrm{~K}$ (Autosorb-6, Quantrachrome). The narrow micropore volume with pore diameter $<0.7 \mathrm{~nm}$ were calculated using the DubininRadushkevich method at relative pressures between 0.0001 and 0.25 from the $\mathrm{CO}_{2}$ adsorption isotherms.

Mercury intrusion porosimetry (PoreMaster, Quantachrome Instruments, USA) was used to calculate the macroporous volume of the activated samples. The total pore volume was hence evaluated as the sum of pore volumes in all PSD ranges.

\subsection{Electrochemical testing}

$6 \mathrm{M} \mathrm{KOH}$ was the electrolyte of choice throughout all electrochemical measurements. An electrochemical interface (Gamry potentiostat, Interface 1000, SciMed, UK) in threeelectrode mode was used for all electrochemical testing. Working electrodes were prepared as follows: $85 \%$ activated carbon, $5 \%$ Super C65 as a booster carbon and $10 \%$ polyvinylidene fluoride (PVDF) as a binder (PI-KEM Ltd., UK) were first mixed in N-methyl-2-pyrrolidone (NMP) solvent (Sigma-Aldrich Ltd, UK). The mixture was then coated on $1 \mathrm{~cm}^{2}$ nickel foam (Suzhou JSD Co. Ltd., China) to form a final active material mass loading of 1-3 mg. The counter and reference electrodes used were platinum mesh and $\mathrm{Ag} / \mathrm{AgCl}$ (Tianjin AiDaHengSheng Technology Development co. Ltd., China), respectively. Galvanostatic charge-discharge (GCD) stability testing was performed at a current density of $20 \mathrm{~A} \mathrm{~g}^{-1}$ over 5000 cycles. Electrochemical impedance spectroscopy was performed over a frequency range 
of $10 \mathrm{mHz}$ to $1 \mathrm{MHz}$ with a voltage amplitude of $5 \mathrm{mV}$, all performed at open circuit potential. The equivalent series resistance, different electronic and ionic contributions of resistive components, constant phase elements and relaxation times of different porous structures were successively determined. The correlation between different morphologies and the electrochemical performance was hence inferred for different porous electrode materials by employing the equivalent circuit model suggested in this study (Echem Analyst software, Gamry Instruments). The variation in specific capacitance before vs. after cycling was also studied.

\section{Results and Discussion}

\subsection{Surface morphology, pore volume and pore size distribution}

SEM and TEM characterization techniques illustrate the etching effect of $\mathrm{KOH}$ on the carbon precursors. The morphology dramatically changes from a fibrous structure with micropores at low $\mathrm{KOH} /$ cellulose ratios to a hierarchical porous network of micro-, mesoand macropores at medium loadings $(\mathrm{KOH}-0.5$ and $\mathrm{KOH}-1)$. Further increase in the $\mathrm{KOH}$ loading enhances the formation of meso- and macropores that dominate at the highest $\mathrm{KOH} /$ cellulose mass ratios. Fig. 1 and S1 show that this change is attributed to the expulsion of the more volatile matter as the $\mathrm{KOH}$ mass was increased, leaving behind a diverse range of pore

diameters. The selection of activation conditions of the carbon precursor with different $\mathrm{KOH}$ loadings determined the width of the PSD in the micro-, meso- and macroporous regions. 

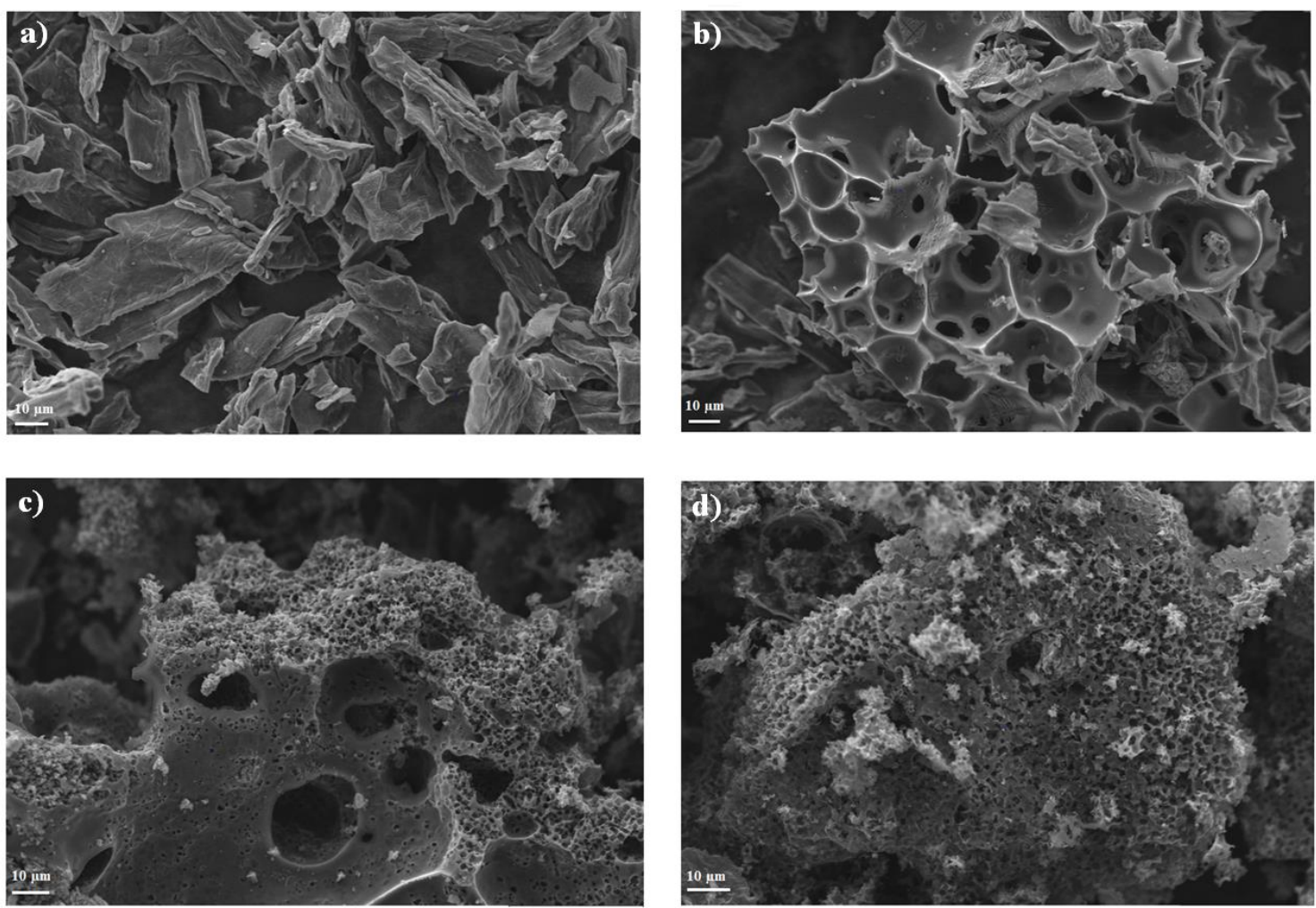

Figure 1: SEM images of four representative activated $\mathrm{KOH}-n$ samples with increasing $n$; a) $\mathrm{KOH}-0.01$, b) $\mathrm{KOH}-0.1, \mathrm{c}) \mathrm{KOH}-0.5$ and d) $\mathrm{KOH}-2$.

The macropore volumes calculated using mercury intrusion porosimetry, and micro- and mesopore volumes determined by the DFT method from $\mathrm{N}_{2}$ and $\mathrm{CO}_{2}$ isotherms, were combined in order to determine the total pore volume in the samples. The dominance of micropores at low or zero chemical activation, and that of macropores at high $\mathrm{KOH}$ concentrations is shown in Fig. 2. The total pore volume increased with the $\mathrm{KOH} /$ cellulose ratio, which was attributed to the formation of a macroporous structure and a decrease in the micropore volume fraction and therefore, contribution to a wider PSD. The pore volume in the range of $0.7-50 \mathrm{~nm}$ displayed sharp peaks in the micro- and mesoporous ranges at different $\mathrm{KOH}$ activation ratios, in addition to the dominance of macropore peaks, whilst still maintaining a mesoporous structure for higher activation ratios (Fig. S2). The volume of $\mathrm{CO}_{2}$ adsorbed also confirms an increase in the total microporous volume (micropores of 
diameter $<0.7 \mathrm{~nm}$ ) with the increasing $\mathrm{KOH}$ loading (Fig. S3). The total pore volume, constituting micro-, meso- and macropores, increased with increasing $\mathrm{KOH}$ loading, as detected by $\mathrm{CO}_{2}$ and $\mathrm{N}_{2}$ isotherms and MIP. However, the micropore fraction decreased with higher $\mathrm{KOH}$ etching due to the formation of more macropores.

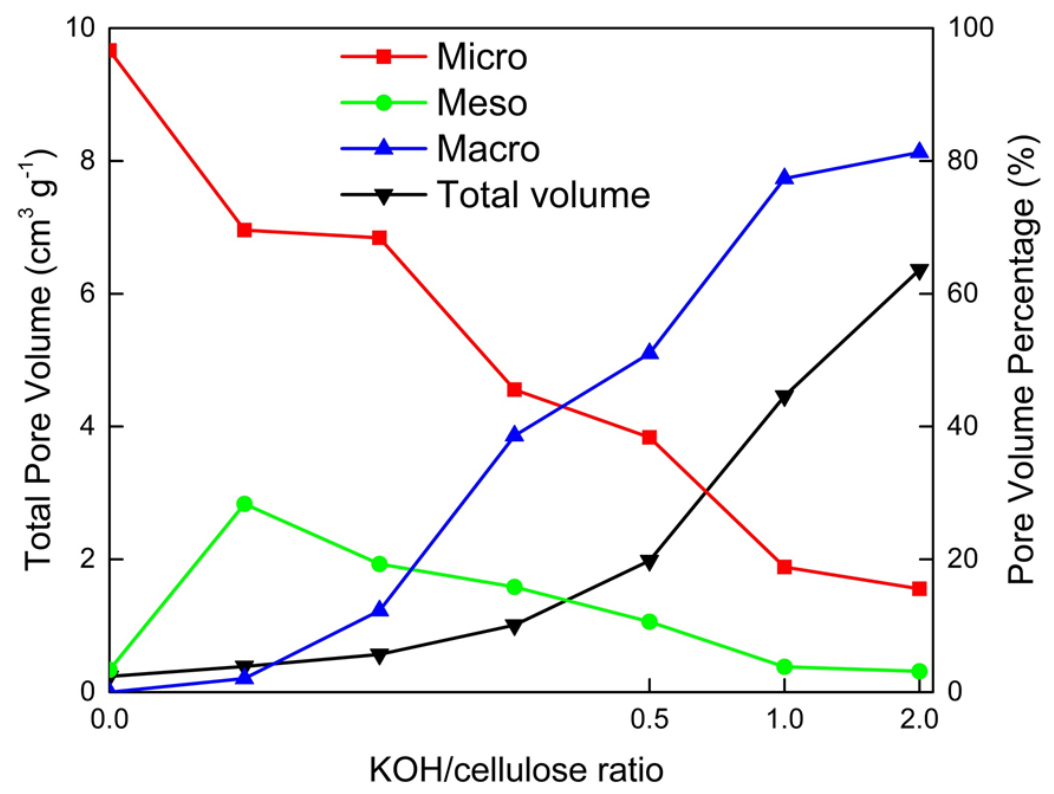

Figure 2: Total pore volume and volume percentage contributions of each of the pore size ranges in different $\mathrm{KOH}$-activated carbons.

The oxygen and carbon contents of the activated carbon samples were detected using the XPS technique (Table S1). The different peaks, $\mathrm{C}_{1 \mathrm{~s}}, \mathrm{O}_{1 \mathrm{~s}}, \mathrm{O}_{\mathrm{KLL}}$ oxygen Auger and $\mathrm{C}_{\mathrm{KLL}}$ carbon Auger, are recorded at binding energies of $284 \mathrm{eV}, 532 \mathrm{eV}$ and $983.68 \mathrm{eV}, 1223.68 \mathrm{eV}$, respectively in all spectra (Fig. S4). It is demonstrated that the oxygen abundance increased with the $\mathrm{KOH}$ loading, indicating the presence of more oxygen functional groups on the carbon surface of increasing total pore volume. The effect of the oxygen content on the electrochemical behavior will be further discussed in the following sections.

\subsection{Frequency-dependent impedance characteristics}


The modelling of the frequency response of porous materials using an equivalent circuit has been discussed in the literature, so as to understand the behavior of different elements that contribute to the total resistance and capacitance values of the whole device $[21,22,26,34-$ 36]. However, it is normally assumed that the electrode is ideally polarizable according to Grahame's definition, whereby a change in potential causes solely a flow of charge from the external circuit to the electrode/electrolyte interphase, with no charge flow across the doublelayer [37]. The double-layer is then at electrostatic equilibrium at a specified potential and the ideal polarizability is detected by a $90^{\circ}$ phase angle (current and voltage are out of phase by $90^{\circ}$ ). This performance is demonstrated by a vertical line, parallel to the imaginary part of impedance ( $\left.Z^{\prime \prime}\right)$, on the y-axis of the Nyquist complex plot. In the case of porous materials, and unlike planar electrodes, non-ideal and incompletely polarizable behavior occurs that is frequency-dependent and is distributed over the porous electrode surface in a non-uniform current distribution mode. Hence, when a small alternating voltage signal is imposed to a porous matrix electrode, the behavior is far from ideal, and the double-layer capacitance is dispersed and frequency dependent, whereby different pore sizes 'tune in' after different periods of time (i.e., the narrower the pore, the longer the time or the lower the frequency) $[23,38,39]$.

In the case of the porous $\mathrm{KOH}$-activated model systems used herein, the morphologies with different PSD and oxygen functionalities determine the capacitive behavior in different frequency regions. EIS was applied at the open circuit potential, and the Nyquist plot, equivalent series resistance, modulus of impedance $\left(Z_{m o d}\right)$, and phase angle $(\phi)$ are presented in Fig. 3. 
a)

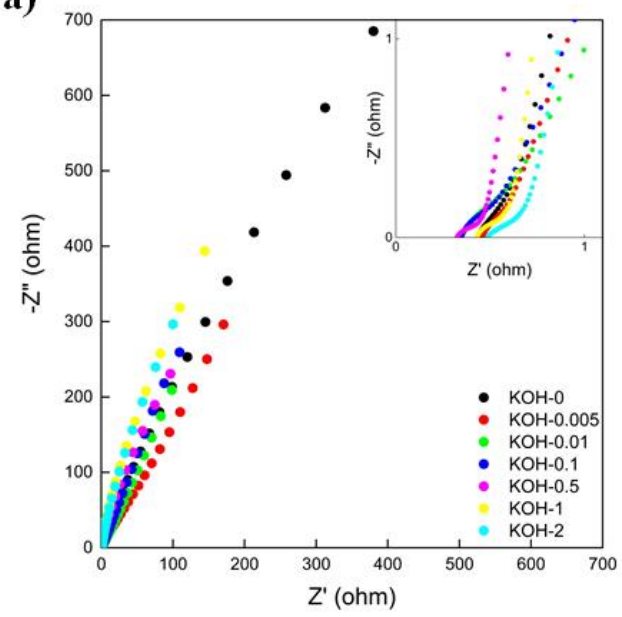

c)

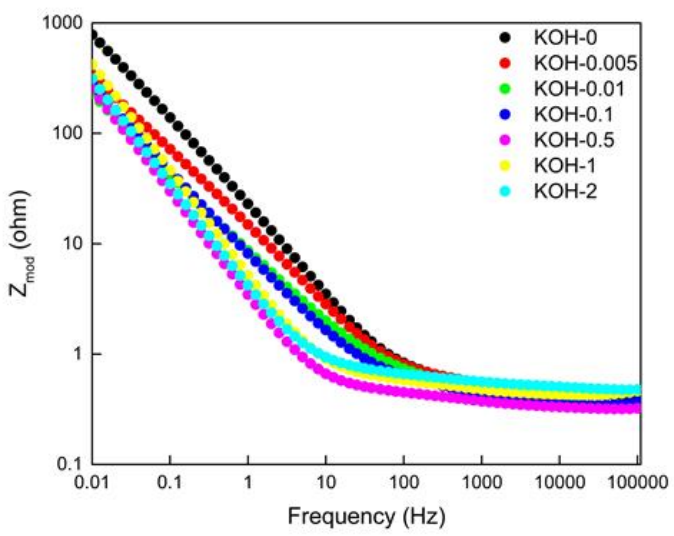

b)

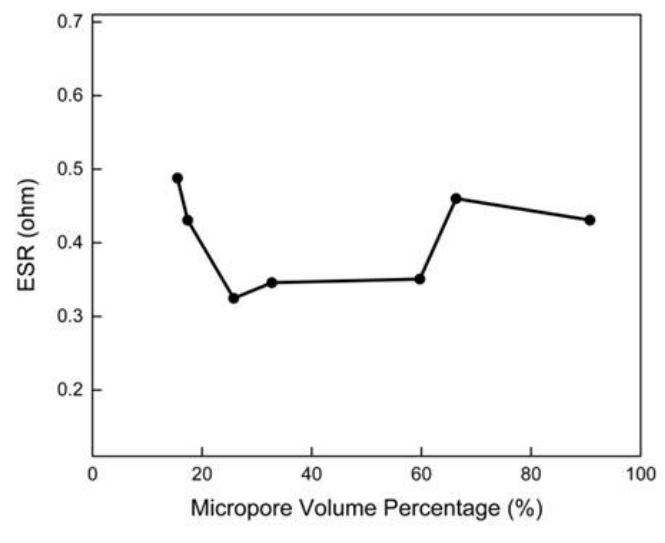

d)

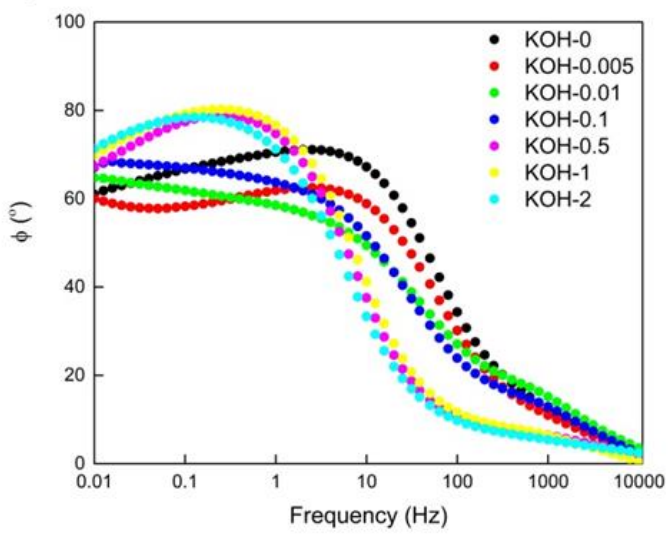

Figure 3: Impedance plots of $\mathrm{KOH}$-activated samples showing: a) Nyquist plot, b) ESR as a function the micropore volume fraction, and Bode plots showing the variation of c) impedance modulus $\left(Z_{m o d}\right)$ with frequency and d) phase angle $(\phi)$ with frequency.

The particular features in order of the speed of processes governing the different resistive and capacitive elements contributing to the electrochemical performance are investigated using the impedance data. As mentioned earlier, the capacitive response is distributed over a wide frequency range, and the examination of the various bulk electrolyte, surface and different pore sizes elements within the carbon electrodes is necessary to understand the impedance behavior. The impedance response will be considered below starting at the highest 
frequencies employed and onto the more complex behavior of the carbon model systems at lower frequencies.

The equivalent series resistance (ESR) represents the impedance behavior at very high frequency $(f \rightarrow \infty)$ and is evaluated at the intercept on the real axis $\left(Z^{\prime}\right)$ of the Nyquist plot (Fig. 3a). This resistance governs the bulk electrolyte ionic resistance and interparticle / intraparticle electrical resistances originating from the porous nature of the electrode materials. The resistance of the bulk electrolyte is the only common component of the ESR in the case of using the same electrolyte media, and therefore the different values attained in the porous carbons reflect the relative change in the internal electrical resistances of the carbon particulates. The ESR value decreased with an increased total pore volume and decrease in the microporous contribution (i.e. with the $\mathrm{KOH}$ loading), where the ESR reached a minimum in $\mathrm{KOH}-0.1$ and $\mathrm{KOH}-0.5$ samples, beyond which the values increase again (Fig. 3b). These results might be attributed to the combination of two different factors. i) A hierarchical porous structure and a balance between the micro- and macropores achieved in $\mathrm{KOH}-0.1$ and $\mathrm{KOH}-0.5$ samples where the micro- and macroporous volume contributions were almost identical, as shown in Fig 2. ii) The increase in the oxygen content in the latter two carbons that optimizes the wettability of these carbons. An increase in oxygenated functional groups of hydrophilic nature can enhance the admittance of electrolyte ions into the smallest pores of the carbon materials.

In the intermediate frequency range, the charge transfer resistance is indicated by the highfrequency semicircle in the Nyquist plots (inset of Fig. 3a). It is clear that not all samples exhibit a distinct semicircle, indicating very low charge transfer resistance due to the lack of a Faradaic contribution associated with redox reactions at the electrode surface. The values slightly decreased with the $\mathrm{KOH}$ loading, which is attributed to the increased accessible 
electrode surface area and hierarchical porous structure that boost the electron/ion transfer in the carbons, despite the increase in their total oxygen content $[3,40]$.

At the lowest frequency of $0.01 \mathrm{~Hz}$, the impedance magnitude $\left(Z_{m o d}\right)$ decreased from the nonactivated sample to activated ones, as the morphology evolved from a fibrous structure to a more porous one (Fig 3c). All of the porous carbons demonstrated a non-ideal performance (phase angle $\phi<90^{\circ}$ ), as shown in the Nyquist plot and Bode plot of the phase angle. The deviation from ideality at low frequencies decreases with the increase in the total pore volume with an increased macroporous contribution (increased $\mathrm{KOH} /$ cellulose mass ratio). This behavior might be associated with the porous network structure comprised of micro-, meso- and macropores that play a major role in determining the response at low frequencies, in which different pore size ranges participate in the impedance response. The wide PSD, dominated by macropores with a high total pore volume, leads to a high diffusion rate of the ions into the pores. Consequently, the formation of macropores eases the access of electrolyte ions into the meso- and micropores, thus constituting a more ideal performance and the phase angles approaching $90^{\circ}$. Whereas the total ultra-micropore volume $(<0.7 \mathrm{~nm})$ was dominant in the low activated samples $(\mathrm{KOH}-0, \mathrm{KOH}-0.005$ and $\mathrm{KOH}-0.01)$, hence impeding the wetting of the finest porous matrices that might not occur even at the lowest frequencies used, leading to non-ideal impedance response [19]. These results will be further investigated in the next section through the evaluation of the specific capacitances using EIS.

The frequency where the phase angle is closest to $45^{\circ}, f_{45}$ (the frequency that represents the transition of prominently resistive to prominently capacitive behavior) also decreases as the total pore volume, with different pore size ranges, is increased with the $\mathrm{KOH}$ loading (Figure 3d). A wider PSD and larger total pore volume at high activation ratios requires longer relaxation times to allow the accessibility of the electrolyte ions into different pore features, starting from macro-, passing into meso- and finally reaching micropores (Fig. 4). Therefore, 
the capacitive character of the electrode will only dominate once the pores are 'tuned in', such that the electrolyte ions can invade the smallest pore sizes.

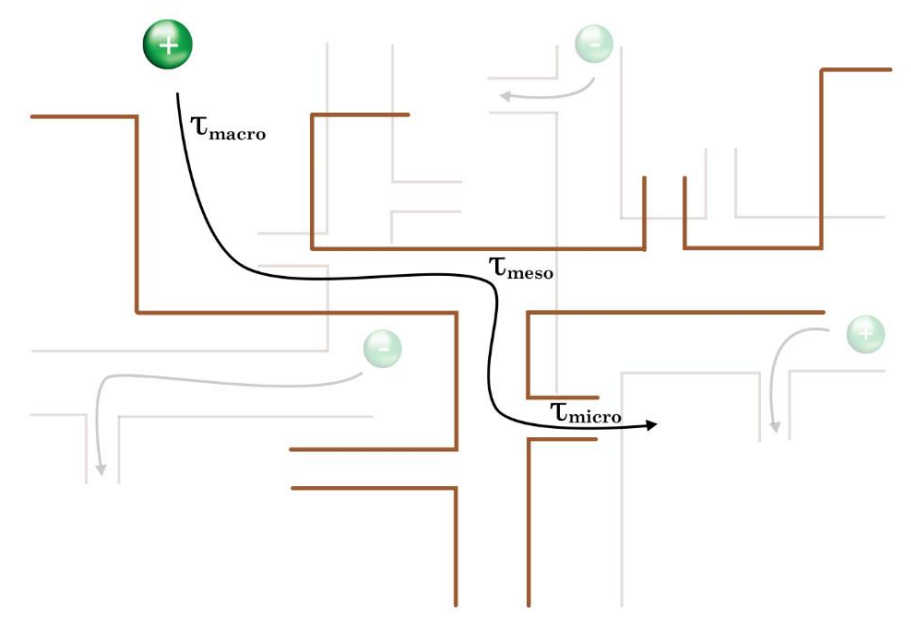

Figure 4: A schematic representation of electrolyte ions transport into the porous electrode, starting with macropores, diffusing into the mesopores and finally reaching the micropores.

\subsection{Equivalent Circuit of Porous Carbon Materials}

Based on the macro/meso/micro pore structure model and the different circuit elements that contribute to the impedance response previously discussed, and using the TLM as a basis, a simple equivalent circuit model is proposed and shown in Fig. 5. The first part of the circuit consists of an inductive element $(L)$ associated with electrical connections; equivalent series resistance $(E S R)$ of the bulk electrolytic, interparticle and intraparticle resistances arising from the porous nature of the electrodes; charge transfer resistance $\left(R_{C T}\right)$ and accompanying constant phase element $\left(C P E_{C T}\right)$. The latter two elements combine to form the semi-circle in the Nyquist plot and model the parallel combination of Faradaic charge transfer resistance and double-layer capacitance at the surface within the porous matrix. A short transmission line is employed using three different time constants $(R C P E)$ that govern the microporous 
$\left(R_{\text {micro }} C P E_{\text {micro }}\right)$, mesoporous $\left(R_{\text {meso }} C P E_{\text {meso }}\right)$ and macroporous $\left(R_{\text {macro }} C P E_{\text {macro }}\right)$ impedance contributions. These series $R C$ combinations represent the migration of ions through the hierarchy of pore sizes that contribute to the total impedance response with different time constants. The resistances described in the transmission line primarily represent the electrolyte resistance in the different pore hierarchies. The capacitance is evaluated inside the pores, effectively modelling the capacitive contribution of each PSD, with corresponding uniform pore size.

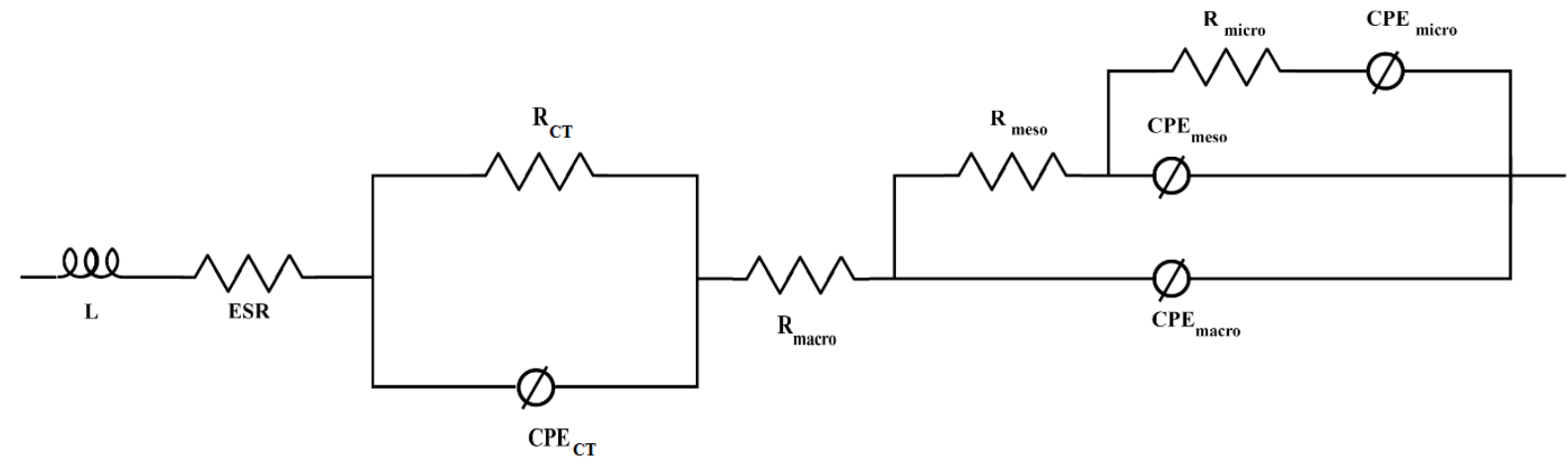

Figure 5: Equivalent circuit of a carbon-based electrode employed in a supercapacitor.

The elements of least resistive nature (i.e. macropores) of the porous matrix are accessed first and hence have the fastest response times at high frequency alternating current or potential step, followed by mesopores, and finally micropores. The parallel (RCPE) network representing the pore contribution to impedance, dominates at low frequencies, whereas the ESR and charge transfer elements govern the EIS behavior at the high frequency limit. This model demonstrates that the current distribution is dispersed non-uniformly (temporally and spatially) in the electrode structure, owing to the inhomogeneities of the porous channels.

The circuit model suggested herein shows a good fit to the impedance responses of the different samples over the wide frequency range employed, whereby the maximum chi- 
square goodness of fit for different impedance behaviors among all the samples is $5.6 \times$ $10^{-2}$ (Fig. S5).

\subsection{Specific capacitance in different frequency ranges}

The "best performance" of electrodes with the highest capacitance values is usually determined from cyclic voltammetry (CV) measurements at a specific sweep rate or by galvanostatic charge-discharge cycles at a specific current density. However, for porous electrodes, the capacitance values are frequency-dependent, and determined using EIS. The change in specific capacitance as a function of frequency for all samples is shown in Fig. 6 and S6, and is calculated as follows [16, 41]:

$$
\begin{gathered}
C^{\prime}(\omega)=\frac{-Z^{\prime \prime}(\omega)}{\omega|Z(\omega)|^{2}} \\
C^{\prime \prime}(\omega)=\frac{Z^{\prime}(\omega)}{\omega|Z(\omega)|^{2}} \\
C(\omega)=C^{\prime}(\omega)-j C^{\prime \prime}(\omega)
\end{gathered}
$$

where $Z^{\prime}, Z^{\prime \prime}$ and $Z$ are the real, imaginary and total impedance values, respectively in ohms, with $j=\sqrt{-1} . C^{\prime}, C^{\prime \prime}$ and $C$ represent the real part, imaginary part and total capacitance, respectively, at a specified frequency, all in Farads. The total capacitance, also known as the equivalent series capacitance, does not have a physical correspondence to the system and its sole purpose is to replicate the frequency response of the system under study [22]. The imaginary part of the capacitance corresponds to the energy dissipation by irreversible processes $[42,43]$. The maximum in the imaginary capacitance-frequency plot corresponds to the dielectric relaxation time of the whole system, in which the frequency response transforms from a resistive to a capacitive one. The results obtained are consistent with the Bode phase plot values, at which $f_{45}$ is evaluated (Fig. S7). The real capacitance part 
represents the characteristic behavior of the capacitance of the electrode structure and the electrode/electrolyte interphase, and is referred to as the gravimetric or volumetric capacitance [44]. To obtain the corresponding real specific capacitances in this instance as a function of frequency, the $C^{\prime}(\omega)$ values were divided by the mass of active material in each of the samples. The specific capacitance values increase with the activation ratio to a maximum in the $\mathrm{KOH}-0.1$ sample, beyond which the capacitance decreases for $\mathrm{KOH}-0.5$ and $\mathrm{KOH}-1$ sample and then again increases with further activation.

The fibrous structure of the non-activated sample evolves into a microporous one in the lowactivated samples, thus increasing the capacitive element of the cumulative number of pores with higher total pore volume. However, further increase of the porous surface area at fixed volume causes an increase in the internal pore resistance with the increased interparticle/intra particle resistances, which might lead to the observed decrease in total capacitance. The carbon matrix composed of micro, meso- and macropores in $\mathrm{KOH}-0.5$ and $\mathrm{KOH}-1$ samples do not exhibit the best performance at low frequency, in which the capacitance is lost due to ineffective electrolyte penetration of the finest pore fraction which itself accounts for most of the specific surface area of these materials. However, for the most activated sample, the macropores dominate the porous network, and the ease of accessibility of the electrolyte ions into the pores leads to an increase in the specific capacitance values again. Thus, it is established that there is a trade-off between the total pore volume, nature and width of the PSD, the specific surface area and the associated impedances within the porous structures. 
a)

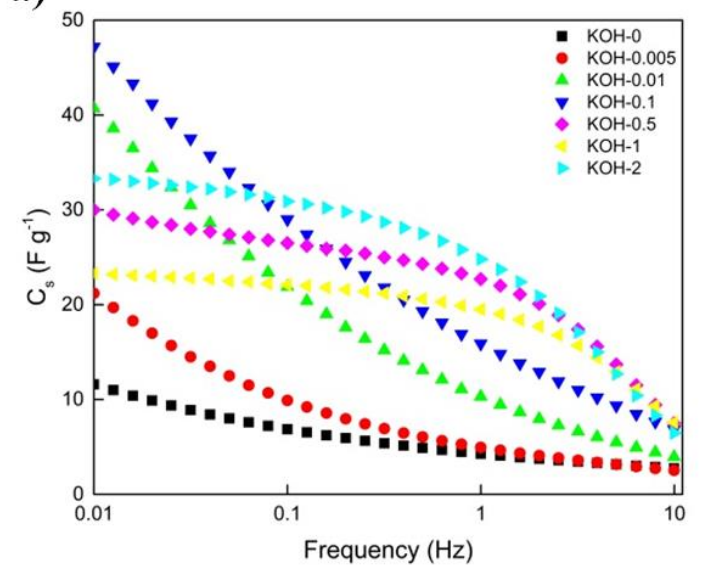

b)

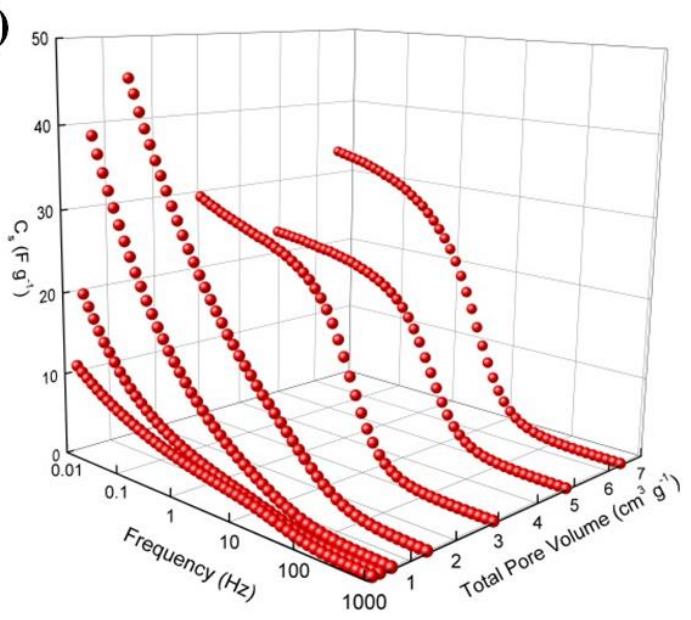

Figure 6: Real part of the specific capacitance of all activated samples in the frequency range of $0.01-10 \mathrm{~Hz}$ demonstrated in a) a Bode plot and b) as a function of frequency and total pore volume determined by $\mathrm{N}_{2}$ and $\mathrm{CO}_{2}$ isotherms and MIP data.

The capacitive behavior in the low-frequency region of $0.01-1 \mathrm{~Hz}$ adopts two different trends amongst the carbons employed as electrode materials: i) a rapid decrease in the low frequency for low-activated samples and ii) a plateau of almost constant capacitance for higher activated samples after which the capacitance declines at higher frequency. These observations suggest that the complete wetting of the finest pore structures has not been reached in the low activated samples and that the capacitance values of these carbons are yet still to increase upon employing frequencies $f<0.01 \mathrm{~Hz}$. This proves that the micropores play a major role in the impedance behavior only at very low frequencies and the performance decays drastically as the time needed for adequate ingress of electrolyte ions inside the fine pores is not sufficient. This is not the case for high-activated samples with high pore volumes, whereby meso- and macropores accommodate a quicker frequency response of the electrolyte solution invasion into bigger pores. The total pore volume increase was accompanied by an increase in the macroporous contribution to the total capacitance value, 
which enhances the performance and quick adaptation of these carbon electrodes at higher frequency responses.

These observations confirm that the determination of best capacitive behavior of porous carbon electrodes in supercapacitors is arbitrary unless evaluated at a specific frequency. Upon applying lower frequency ranges to the low activated samples, the capacitances will further increase to reach a plateau profile similar to that of the high activated samples, indicating maximum utilization of the accessible pores by the electrolyte ions.

These results do not imply that the wettability of the finest pore structures (i.e. ultramicropores) is achieved, which consequently might impede the response behavior and lead to loss of the total capacitances. However, the maximum capacitance values in the low activated samples (KOH-0.01 and $\mathrm{KOH}-0.1)$ would be even higher than those achieved with the carbons of high $\mathrm{KOH}$ loading that have already reached their maximum values (plateau profile), indicating that the low activated materials might be more suitable for applications with very low-frequency responses. It should be also noted that the capacitance values obtained are a function of the mass loading of the active material, whereby increasing the electrode total mass can lead to higher capacitance values, and in some cases slower relaxation times, as will be elaborated in the following section, yet might also increase the equivalent series resistance and electrolyte resistances within an increased number of pores. Thus, the mass loading also plays an important role in analyzing the impedance response and obtaining the intrinsic specific capacitance values if of major importance to analyze the performance optimization of supercapacitor electrode materials. In this work, the mass of deposited electrode material was kept constant amongst the different $\mathrm{KOH}$ activated samples, and the preparation method was consistent throughout all experiments to evaluate the materials' characteristics, independent of different parameters in the preparation process. In addition, all electrode materials were deposited on porous nickel foam, acting as a current 
collector, in which the values deduced from EIS method, might also change with using different current collectors, electrolyte media and/or three-electrode setups.

\subsection{Determination of circuit elements and relaxation times}

The model components discussed in the previous section can reproduce, with high precision, the small voltage amplitude impedance behavior of the carbon electrodes over a wide frequency range. Pores in the same size range are assumed to be identical with a uniform cross-section, and the curvature and surface roughness of pores are neglected. Furthermore, in each of the $R C P E$-series elements in the transmission line, the capacitance and electrolyte resistance inside the pores is considered to be independent of the distance from the orifice of the pore down its length. It is worth mentioning that other equivalent circuits can be proposed with random sets and different values of $R C P E$ elements, which can generate similar fittings, yet have no correspondence to the real physical values of the different element components [22].

The time constant of each $R C P E$ series combination is calculated as the product of the nonideal capacitance and the sum of resistances from the element being evaluated inward to the pore surfaces and channels from the outer electrode/bulk solution interphase, as follows:

$$
\begin{gathered}
\tau_{\text {macro }}=C P E_{\text {macro }}\left(E S R+R_{C T}+R_{\text {macro }}\right) \\
\tau_{\text {meso }}=C P E_{\text {meso }}\left(E S R+R_{C T}+R_{\text {macro }}+R_{\text {meso }}\right)
\end{gathered}
$$

and $\quad \tau_{\text {micro }}=C P E_{\text {micro }}\left(E S R+R_{C T}+R_{\text {macro }}+R_{\text {meso }}+R_{\text {micro }}\right)$

where

$$
C P E_{i}=C^{\alpha} \quad \text { with } \alpha \text { accounting for non-ideality }
$$

The capacitive elements of the smallest pore dimensions, i.e. the micropores, have the highest time constants and therefore the slowest response rate at low frequencies $(<0.1 \mathrm{~Hz})$, followed 
by mesopores and finally macropores [19]. Consequently, the change in the imposed electrode potential does not charge or discharge the different areas of the matrix at a uniform rate; in reality, the time constants can vary by orders of magnitude $[19,22]$. The asynchronous or non-uniform charging at different rates (or discharging behavior) of a porous electrode hence constitutes a far more complex impedance behavior than for planar electrodes with ideal performance. This leads to the conclusion that the concept of a "best capacitive" behavior cannot be inferred unless specified at a certain frequency.

a)

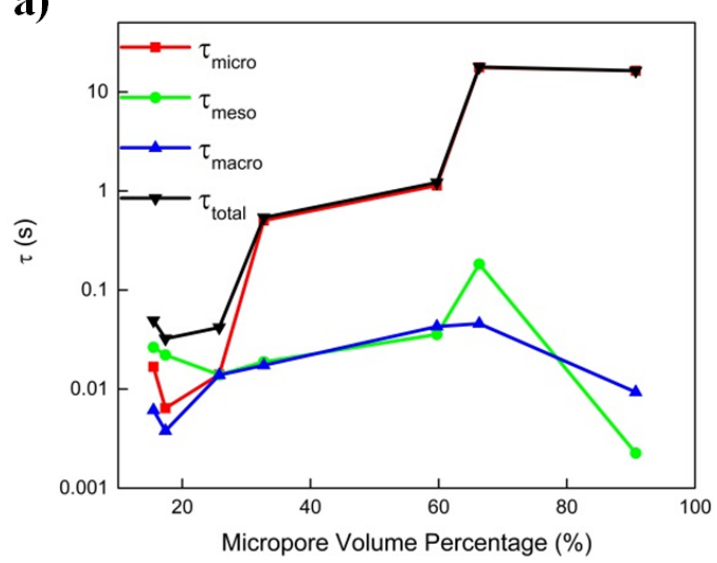

b)

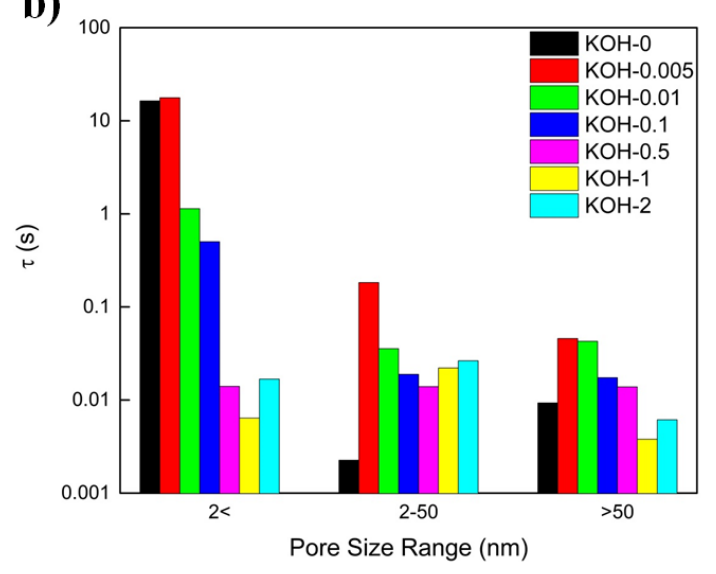

Figure 7: Variation of the relaxation times in all activated carbons as a function of a) the micropore volume percentage and b) with the $\mathrm{KOH}$ loading with different total pore volumes in each PSD.

The relationship between the morphology of the $\mathrm{KOH}$-activated porous carbons and the relaxation times is shown in Fig. 7. It is clear that the microporous volume fraction decreases, while that of macropores increases with $\mathrm{KOH} /$ cellulose ratio, as demonstrated with the structural characterization in the previous sections. In addition, the total pore volume was illustrated to increase with macropore volume contribution. The results obtained demonstrate 
a co-dependence between the nature of the PSD, the total pore volume and the total relaxation times evaluated for the different porous carbons.

Upon increasing the $\mathrm{KOH}$ activation loading from the non-activated sample with a dominant ultra-microporous structure $(\mathrm{KOH}-0)$ to the lowest activated one $(\mathrm{KOH}-0.005)$, an increase in the total relaxation time, as well as the relaxation times of each of the porous structures, occur. This is due to the introduction of chemical activation that leaves behind a limited porous structure upon carbonization in inert atmospheres. However, a further increase in the activation ratio, and thus an increase in the total pore volume with macroporous morphologies, leads to a decrease in the total and PSD-specific relaxation times, whereby meso- and macro-pores have a lower resistive component compared to micropores in the transmission line. These values are also corroborated by the decreasing impedance magnitude upon increasing the $\mathrm{KOH}$ loading. Beyond an optimum activation loading in sample $\mathrm{KOH}-1$, in which the micro- and macro-pores both contribute to the frequency response, the impedance magnitude increases again in $\mathrm{KOH}-2$ sample (Fig. 3a). This is attributed to the further increase in total pore volume, wherein although the micropore fraction has decreased, the total number of the pores in different size ranges has drastically increased. This leads to the increase in the total relaxation charging/discharging times of $\mathrm{KOH}-2$ electrode. The formation of a more meso- and macro-porous structure in a high total pore volume serves to increase the total relaxation times that are needed for adequate access of the electrolyte ions into the increased number of micropores. This is demonstrated in Fig. $7 \mathrm{~b}$ which shows the different relaxation times in each pore size range of the carbonaceous samples.

The difference in order of magnitudes between the different PSD relaxation times at low or no activation for the same sample diminishes as the activation is further increased, whereby all PSDs contribute significantly to the total time periods needed to charge or discharge the supercapacitor. The frequency response of different pores thus becomes more homogenous as 
a balance between the micro- and macroporous contributions to the total pore volume is achieved. The employment of lower frequencies for the low activated samples will further increase the total relaxation times with the increase of the microporous relaxation times with the micropores requiring the longest periods of time to charge/discharge. Consequently, the difference in order of magnitudes of the PSD-specific relaxation times will further escalate due to the employment of the meso- and macropores at higher frequencies. This is not the case for high activated samples, as the capacitances have reached their maximum values with the plateau character, as previously shown, implying the full utilization of the porous matrices upon applying frequencies as low as $0.01 \mathrm{~Hz}$. These assumptions are also validated with the Bode plots of the imaginary part of the specific capacitances, analyzed in the previous section. The imaginary capacitance, $\mathrm{C}$ '(w) is ascribed to the energy loss by irreversible processes, in which its values demonstrate an increase with the $\mathrm{KOH}$ loading to a maximum in $\mathrm{KOH}-0.01$ and $\mathrm{KOH}-0.1$ samples, beyond which it decreases again. The energy loss has thus increased with the increase in the imaginary capacitance values, due a redistribution of the charges within the pores in non-uniformly charge porous electrodes requiring lower frequencies and thus slow relaxation times. A porous electrode with transmission line model behavior (or truncated one) subjected to a charging regime of period shorter than the RC time constant, will be incompletely charged, whereby the inner regions and smaller pores will be less charges that the outer surface regions and bigger pores. Upon terminating the charging period, the redistribution of the charges in the porous matric starts to achieve a uniform voltage across the matrix, causing a potential decline on the outer surface and thus an apparent energy loss. The extension of the impedance measurement at lower frequencies will demonstrate higher relaxation times for the low activated samples, and consequently higher energy dissipation values associated with the electrical relaxation process. 
These observations thus dictate the accommodation of different carbon materials for designated power applications. Porous structures that can quickly accommodate the charging/discharging behavior, similar to the high activated $\mathrm{KOH}$ sample models, can be employed in applications that require high-frequency responses without major losses in the capacitive mechanism. Whereas carbon materials that are capable of delivering high capacitances but require slow charging/discharging rates, such as the low activated carbons used herein, can be employed in applications of low-frequency response demands.

The suggested model and extracted values of impedance and relaxation times confirm that when addressing a porous matrix with a small alternating voltage signal, the impedance behavior is far from ideal, and is not uniformly accessible by the electrolyte solution.

\subsection{Comparison with 1-PSD electrode equivalent circuit}

The equivalent circuit suggested in this work takes into account three distinct PSDs (micro-, meso- and macro-PSDs), to which an average pore size range can be assigned to each. The different elements, as discussed earlier, represent different resistive and capacitive components in the circuit. However, if it was assumed that the porous matrices were homogenous in the carbonized samples and composed of one uniform pore size, a more simplified circuit can be used to describe the impedance behavior. This circuit would then be composed of the inductive $L$, equivalent series resistance $E S R$, constant phase element $C P E_{1}$ and charge transfer resistance $R_{C T}$ elements, similar to the previously suggested circuit, but with only one capacitive component $\left(C P E_{2}\right)$ that describes the capacitance inside the pores (Fig. 8a). The relaxation time for $C P E_{2}$ would then be calculated as follows:

$$
\tau_{C P E_{2}}=C P E_{2}\left(E S R+R_{C T}\right)
$$


The relaxation time for one PSD model is compared to the total relaxation time of the truncated version of the TLM suggested in this work (Fig. 8b). The difference between the values is significant for the low- and non-activated samples, where the total pore volume is a minimum and composed primarily of micropores. The matrix nature of these samples was mainly composed of micropores and the PSD-specific relaxation times varied by an order of magnitude. In this case, one capacitive element of the porous matrix cannot govern the impedance behavior of such a PSD. Further activation of the carbon materials leads to a decrease in the percentage difference of the relaxation times for the same carbon material, wherein the porous structure is composed of more macropores and attains a greater porous structure. As the microporous structure develops to a more meso- and macro-porous one, the variance between both models was still high, yet decreased by an order of magnitude on account of the similarity in the frequency response (and relaxation times) of the pores at high activation ratios. 


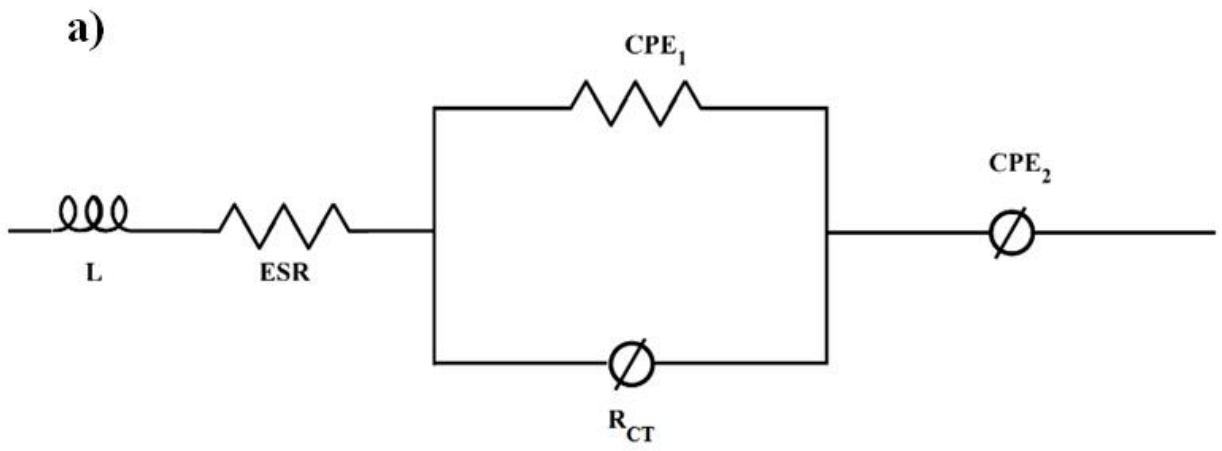

b)

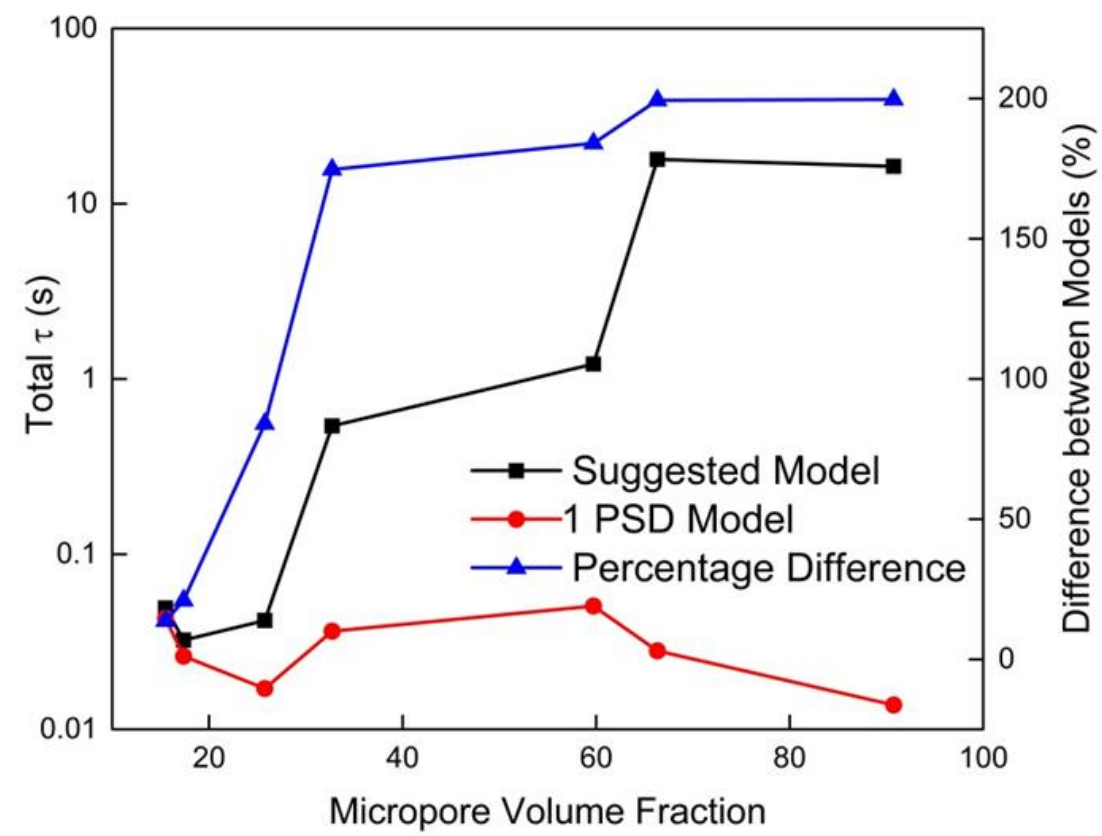

Figure 8: a) Equivalent circuit of porous materials with one PSD and b) the difference between the one PSD model and proposed model as a function of the total pore volume determined by BET.

\subsection{Effect of cycling on EIS and specific capacitance}

The effect of the PSD on the capacitance values at the lowest frequency employed was examined before and after cycling the different electrode materials for 5000 cycles at $20 \mathrm{~A} \mathrm{~g}^{-}$

1. The specific capacitance values obtained from EIS measurements at $0.01 \mathrm{~Hz}$, before and 
after cycling, are shown in Fig. 9. The lowest frequency was chosen to evaluate the capacitance values to include the contribution of the different PSD capacitive elements to the total electrochemical behavior. The capacitance retention rates are usually reported to be less than $100 \%$ in which indicated values do not correspond to the frequency-dependant region [45-47]. However, depending on the porous structure tested, different results can be obtained. For low-activated materials, the specific capacitance increased upon cycling. This is due to the high volume fractions of micropores that dominate the porous network. Upon cycling, the electrolyte can penetrate parts of the structure that were not accessible by electrolyte ions prior to cycling. The pore fractions in the porous matrix can then be slowly wetted by the electrolyte, whereby the smallest pores account for the highest capacitance values, and hence the increase in overall specific capacitance after 5000 cycles [48-50]. This is not the case for the $\mathrm{KOH}-0.5, \mathrm{KOH}-1$ and $\mathrm{KOH}-2$ samples where the meso- and macropores were accessed by electrolyte ions when running impedance measurements prior to cycling, and hence a decay in performance after 5000 cycles is observed. The complete employment of the pores as capacitive elements hence comes into effect at different rates depending on the PSD of the electrode material. These results agree with the trends of specific capacitances demonstrated in Fig. 6, where the rapid decrease in the specific capacitance for low-activated samples was dissimilar to the plateau capacitive profile for high activated samples. This was also demonstrated in the phase angle values, whereby the rapid capacitive-to-resistive transition for the low-activated samples before pore exploitation is opposed to extended capacitive performance for higher activated samples in which the pores are more easily accessed through the hierarchical structure. The behavior recorded upon cycling the electrode materials is accompanied by an increase in the impedance in the low-frequency region for high-activated samples and vice-versa for the low-activated ones, noting that both samples had smaller deviation from ideality upon cycling (Fig. S8). 


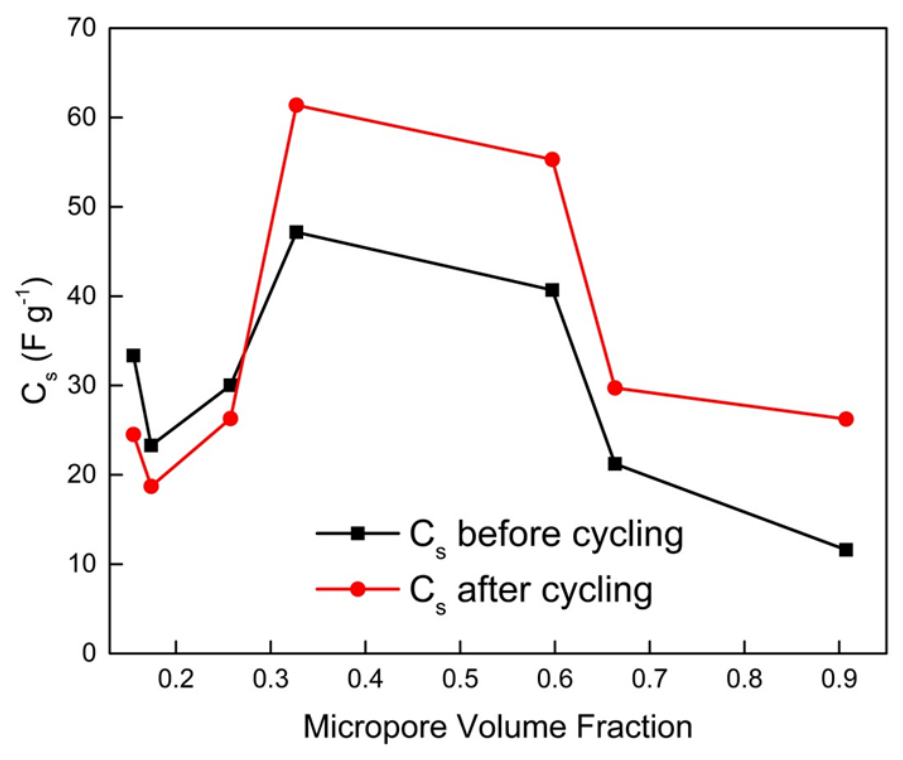

Figure 9: Specific capacitances for all activated carbon materials before $v s$. after cycling as a function of the microporous volume fraction determined by BET.

The specific capacitance values reported throughout this work reflect the electrode materials properties on the microscopic level to further understand the behavior of porous carbon materials in supercapacitors. However, this is the first step in the optimization process from a form-function perspective, and the integration of these realizations with device optimization should be considered for industrial and scale-up applications. In another work, we reported that the supercapacitor configuration and packaging is of major importance, and that different device properties can hugely influence the energy and power delivery of supercapacitors [51]. The understanding and optimization of the synergy between microscopic materials properties and macroscopic device architecture should therefore be implemented for optimized performances of real supercapacitor devices.

\section{Conclusions}

A truncated transmission line equivalent circuit model has been designed and implemented in three-electrode systems for carbon-based supercapacitors. This model explains the different 
capacitive and resistive elements over a wide frequency response. The effect of a wide pore size distribution on the impedance behavior is examined and introduced in the equivalent circuit for the first time. A 'best performing' supercapacitor device cannot be quantified without specifying the nature of the capacitance reported and its frequency dependency. The amount of micropores plays a vital role in the specific surface area and total capacitance, but unless effectively entered by electrolyte ions, a loss in capacitance occurs after long-term cycles. Meso- and macropores accommodate the change in an alternating voltage signal more quickly, and hence such porous structures can be implemented in applications that require a high response rate. The present work demonstrates that an assessment of the relaxation times is essential to demonstrate the trade-off and possible optimization of porosity, pore size distribution, internal pore resistance and capacitance. The evaluation of these parameters of different porous structures at different rates of frequency response for a supercapacitor device can then be studied to assess power capabilities in charging and discharging and thereby its application for real-world applications.

\section{Acknowledgements}

The authors acknowledge the funding from the Engineering and Physical Sciences Research (EPSRC) under grants (EP/G036675/1; EP/L015862/1) and the Royal Academy of Engineering for support of PRS. The cellulose raw materials were kindly provided by UPMKymmene Corporation. 


\section{Supporting Information}
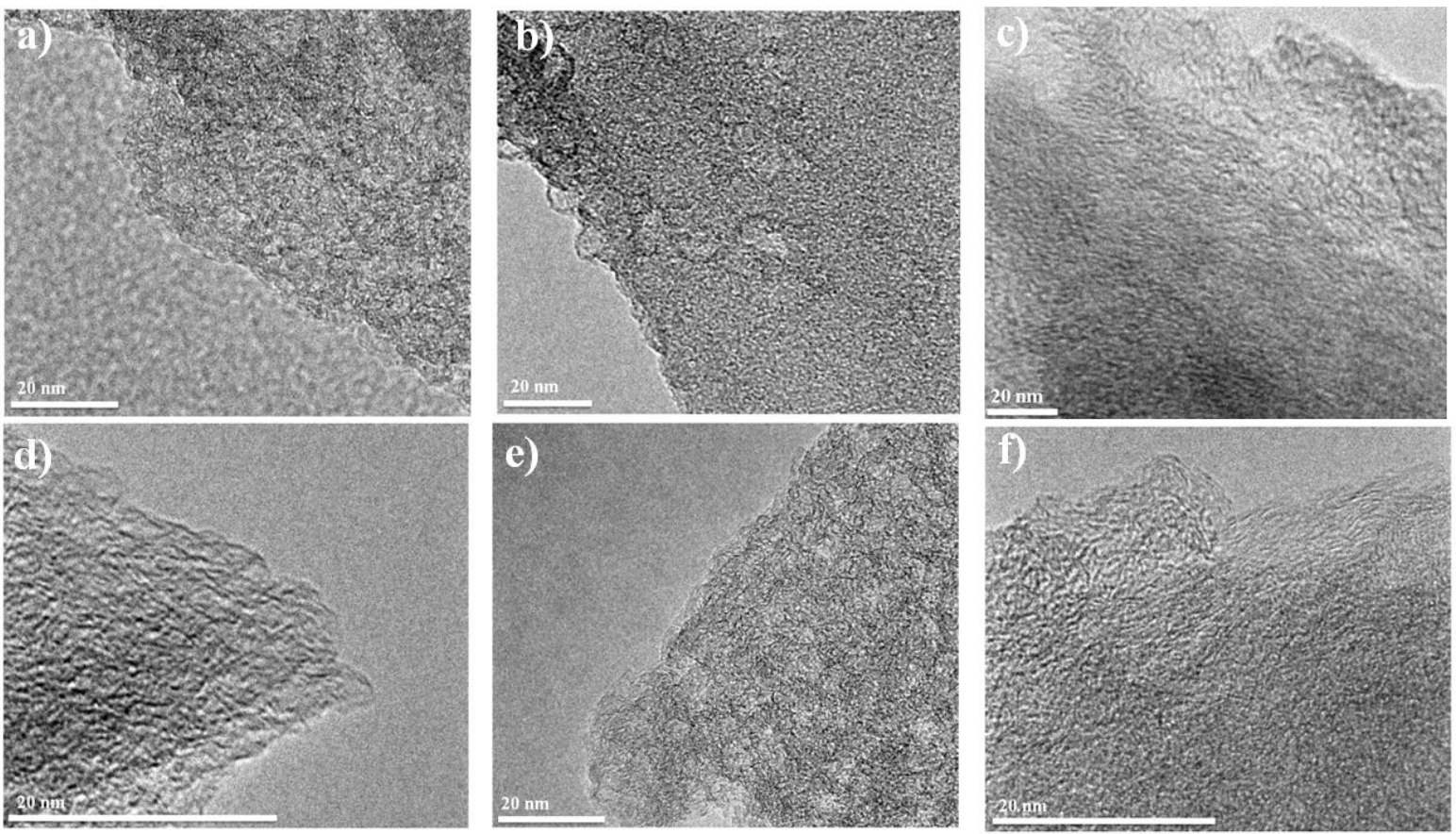

Figure S1: TEM images of various activated $\mathrm{KOH}-n$ samples with increasing order of $n$ : a)

$\mathrm{KOH}-0.005$, b) $\mathrm{KOH}-0.01$, c) $\mathrm{KOH}-0.1$, d) $\mathrm{KOH}-0.5$, e) $\mathrm{KOH}-1$ and f) $\mathrm{KOH}-2$.

a)

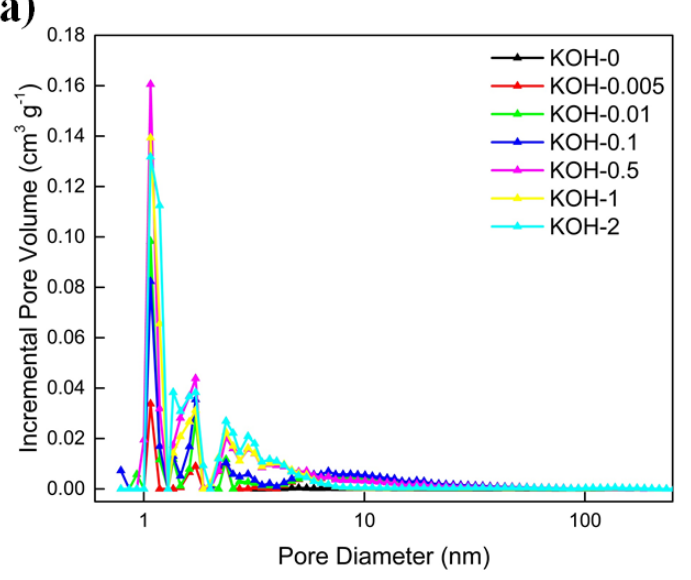

b)

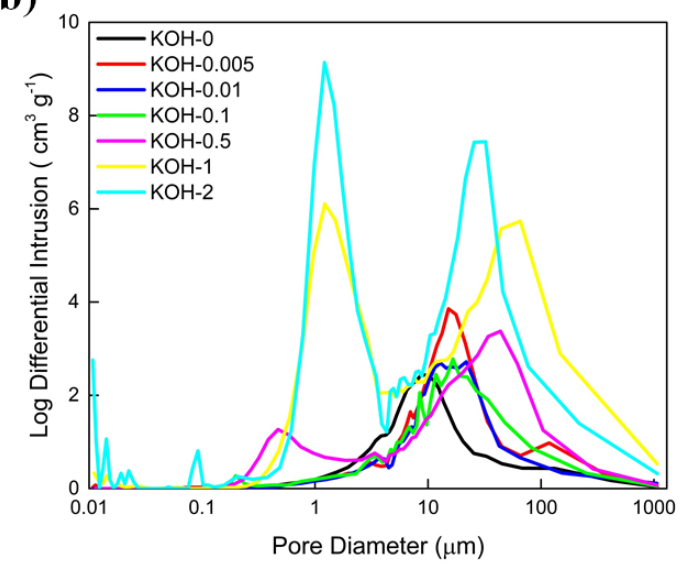


Figure S2: PSDs of $\mathrm{KOH}-n$ activated carbons in the a) micro- and mesoporous ranges obtained by DFT methods from nitrogen adsorption isotherms and b) macroporous region obtained by mercury intrusion porosimetry.

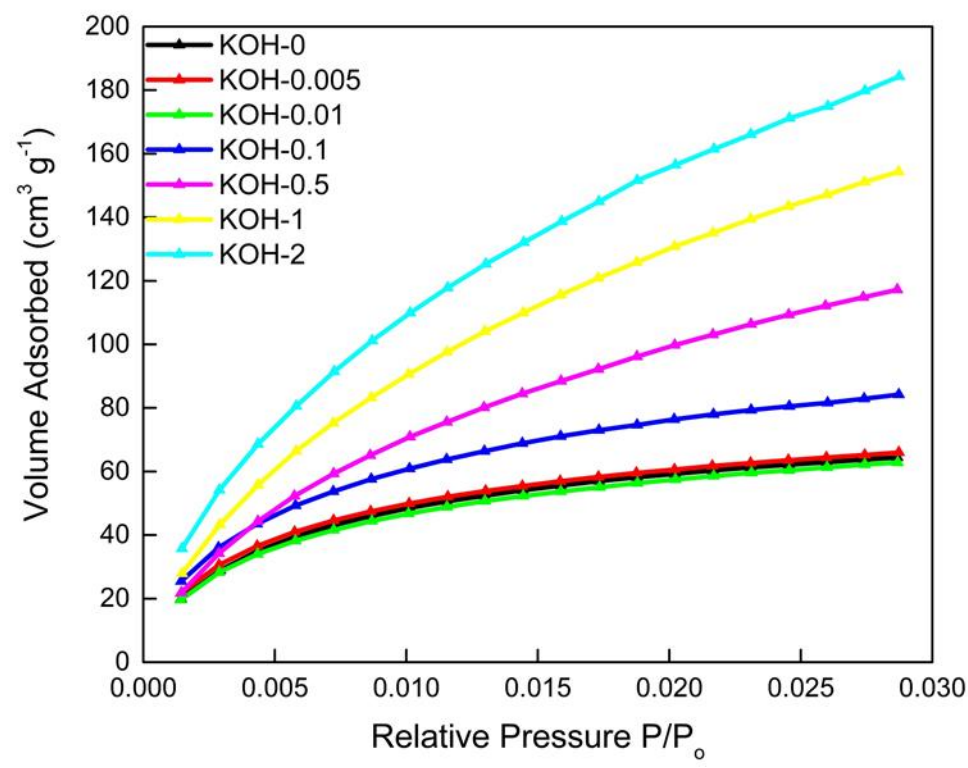

Figure $\mathrm{S} 3: \mathrm{CO}_{2}$ isotherms of $\mathrm{KOH}-n$ samples.

Table S1: XPS data of all activated carbons.

\begin{tabular}{lccccc}
\hline Sample & O:C Ratio & $\mathrm{C}-\mathrm{C}, \mathrm{C}=\mathrm{C}, \mathrm{C}-\mathrm{H}$ & $\mathrm{C}-\mathrm{O}$ & $\mathrm{C}=\mathrm{O}$ & $\mathrm{O}-\mathrm{C}=\mathrm{O}$ \\
& $(\%)$ & $(284.1-284.2 \mathrm{eV})$ & $(285.7-285.9 \mathrm{eV})$ & $(287.1 \mathrm{eV})$ & $(288.4-288.8 \mathrm{eV})$ \\
\hline $\mathrm{KOH}-0$ & 7.78 & 77.6 & 12.6 & 5.1 & 4.7 \\
$\mathrm{KOH}-0.005$ & 9.6 & 77.0 & 12.7 & 5.6 & 4.7 \\
$\mathrm{KOH}-0.01$ & 14.1 & 75.5 & 14.6 & 5.4 & 4.5 \\
$\mathrm{KOH}-0.1$ & 21.11 & 70.3 & 20.6 & 2.7 & 6.4 \\
$\mathrm{KOH}-0.5$ & 21.01 & 67.9 & 21.1 & 5.9 & 5.1 \\
$\mathrm{KOH}-1$ & 18.06 & 65.2 & 21.2 & 7.6 & 6.0
\end{tabular}




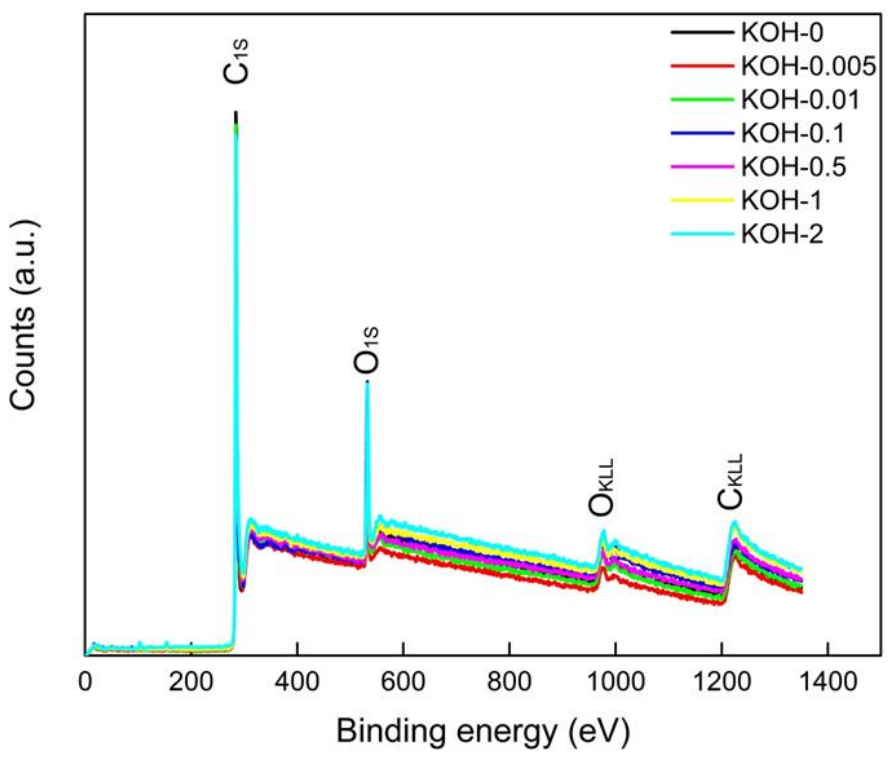

Figure S4: XPS spectra of all activated samples. All spectra show C1s, O1s, oxygen Auger (OKLL) and carbon Auger (CKLL) peaks at binding energies of $284 \mathrm{eV}, 532 \mathrm{eV}$ and 983.68 $\mathrm{eV}, 1223.68 \mathrm{eV}$ respectively. 


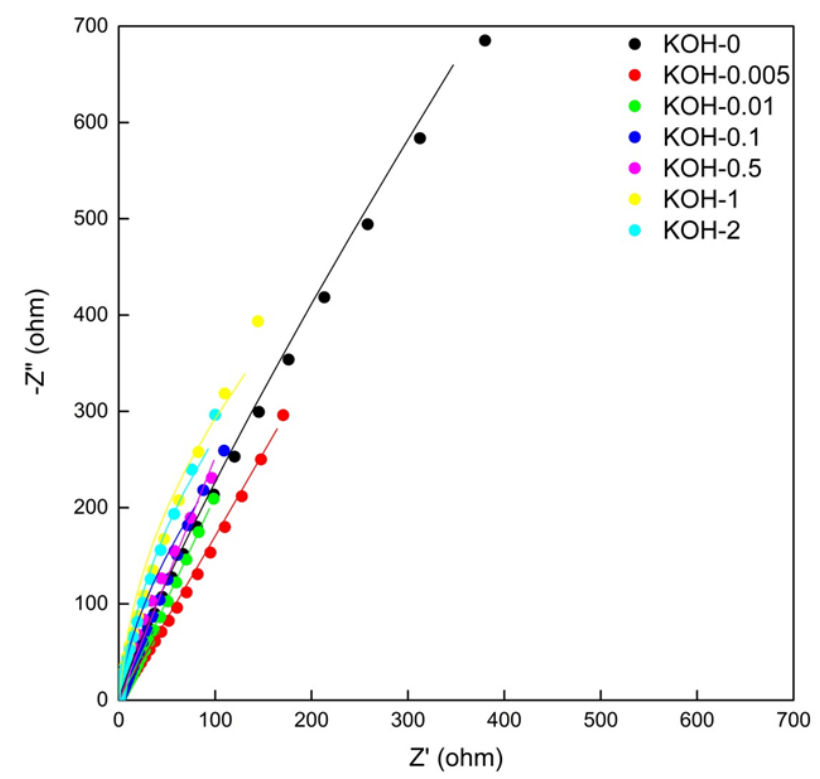

Figure S5: Nyquist plots of all $\mathrm{KOH}$-activated samples with fitting curves according to the equivalent circuit suggested in this work. Chi-square Goodness of fit has maximum value of $5.6 \times 10^{-2}$.

a)

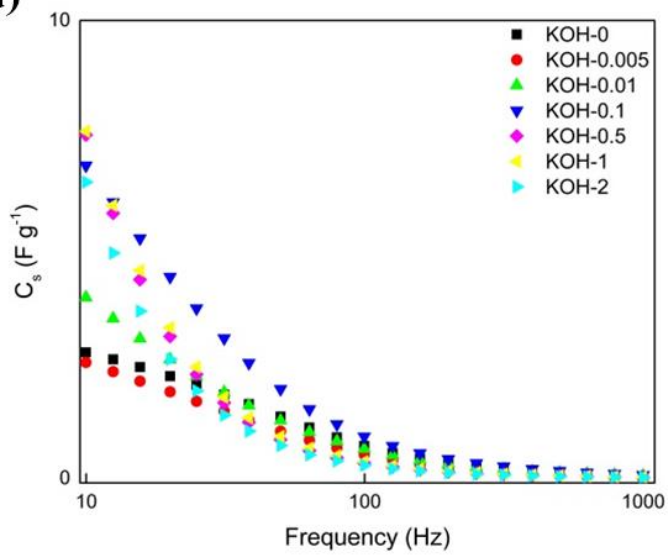

b)

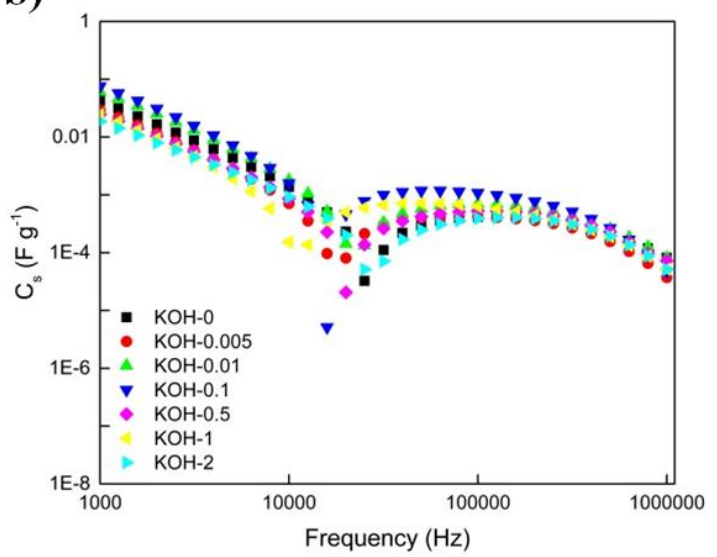

Figure S6: Bode plots of real specific capacitance values of all samples in the frequency range of a) $10-1000 \mathrm{~Hz}$ and b) $1000 \mathrm{~Hz}-1 \mathrm{MHz}$. 


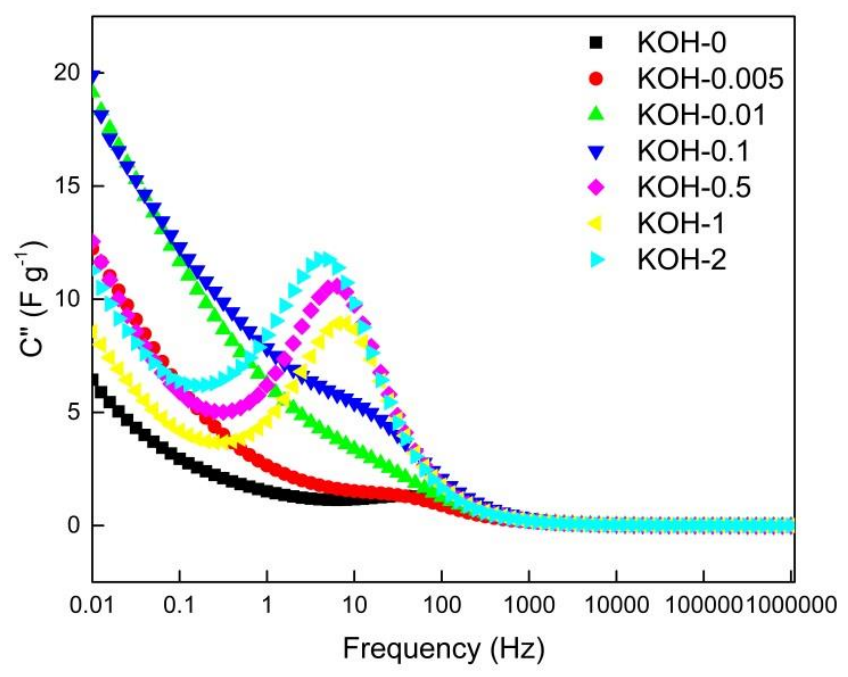

Figure S7: Imaginary part of the specific imaginary capacitance over the studied frequency range.

a)

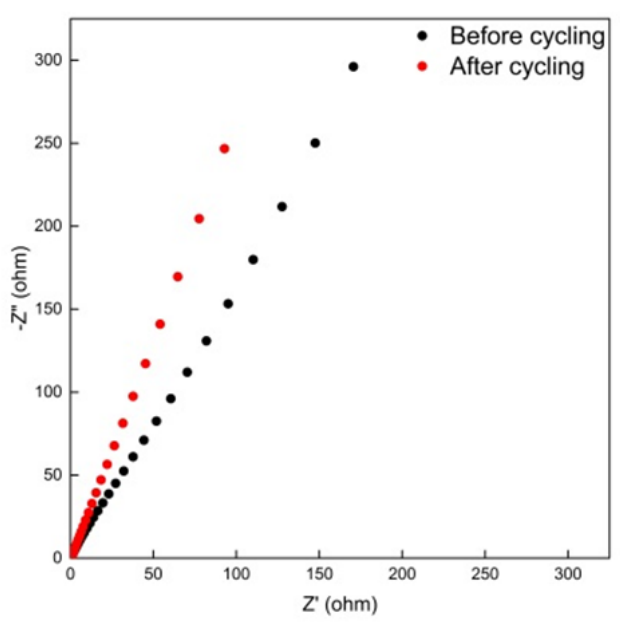

b)

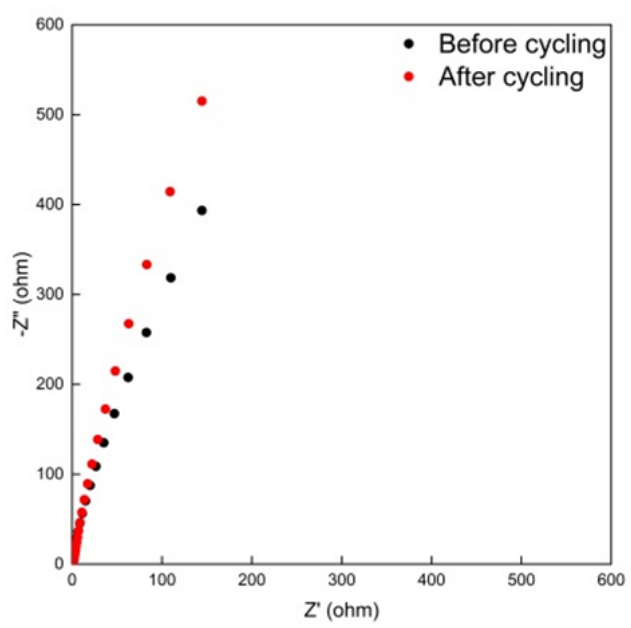

Figure S8: Nyquist plots showing the difference in impedance behavior before cycling and after 5000 cycles for a) $\mathrm{KOH}-0.005$ and b) $\mathrm{KOH}-1$ samples. 



\section{References}

[1] E. Frackowiak, F. Béguin, Carbon materials for the electrochemical storage of energy in capacitors, Carbon, 39 (2001) 937-950.

[2] L.L. Zhang, X. Zhao, Carbon-based materials as supercapacitor electrodes, Chemical Society Reviews, 38 (2009) 2520-2531.

[3] A.G. Pandolfo, A.F. Hollenkamp, Carbon properties and their role in supercapacitors, Journal of Power Sources, 157 (2006) 11-27.

[4] P.L. Taberna, C. Portet, P. Simon, Electrode surface treatment and electrochemical impedance spectroscopy study on carbon/carbon supercapacitors, Applied Physics A, 82 (2006) 639-646.

[5] C. Niu, E.K. Sichel, R. Hoch, D. Moy, H. Tennent, High power electrochemical capacitors based on carbon nanotube electrodes, Applied Physics Letters, 70 (1997) 1480-1482.

[6] C. Vix-Guterl, E. Frackowiak, K. Jurewicz, M. Friebe, J. Parmentier, F. Béguin, Electrochemical energy storage in ordered porous carbon materials, Carbon, 43 (2005) 1293-1302.

[7] H. Shi, Activated carbons and double layer capacitance, Electrochimica Acta, 41 (1996) 16331639.

[8] G. Salitra, A. Soffer, L. Eliad, Y. Cohen, D. Aurbach, Carbon Electrodes for Double-Layer Capacitors I. Relations Between Ion and Pore Dimensions, Journal of the Electrochemical Society, 147 (2000) 2486-2493.

[9] Y. Lu, S. Zhang, J. Yin, C. Bai, J. Zhang, Y. Li, Y. Yang, Z. Ge, M. Zhang, L. Wei, M. Ma, Y. Ma, Y. Chen, Mesoporous activated carbon materials with ultrahigh mesopore volume and effective specific surface area for high performance supercapacitors, Carbon, 124 (2017) 64-71.

[10] J.Y. Hwang, M. Li, M.F. El-Kady, R.B. Kaner, Next-Generation Activated Carbon Supercapacitors: A Simple Step in Electrode Processing Leads to Remarkable Gains in Energy Density, Advanced Functional Materials, 27 (2017) 1605745-n/a.

[11] C. Wei, J. Yu, X. Yang, G. Zhang, Activated Carbon Fibers with Hierarchical Nanostructure Derived from Waste Cotton Gloves as High-Performance Electrodes for Supercapacitors, Nanoscale Research Letters, 12 (2017) 379.

[12] D.I. Abouelamaiem, G. He, I. Parkin, T.P. Neville, A.B. Jorge, S. Ji, R. Wang, M.-M. Titirici, P.R. Shearing, D.J.L. Brett, Synergistic relationship between the three-dimensional nanostructure and electrochemical performance in biocarbon supercapacitor electrode materials, Sustainable Energy \& Fuels, (2018).

[13] D. Zhou, H. Wang, N. Mao, Y. Chen, Y. Zhou, T. Yin, H. Xie, W. Liu, S. Chen, X. Wang, High energy supercapacitors based on interconnected porous carbon nanosheets with ionic liquid electrolyte, Microporous and Mesoporous Materials, 241 (2017) 202-209.

[14] M. Oschatz, S. Boukhalfa, W. Nickel, J.P. Hofmann, C. Fischer, G. Yushin, S. Kaskel, Carbidederived carbon aerogels with tunable pore structure as versatile electrode material in high power supercapacitors, Carbon, 113 (2017) 283-291.

[15] A. Celzard, F. Collas, J.F. Marêché, G. Furdin, I. Rey, Porous electrodes-based double-layer supercapacitors: pore structure versus series resistance, Journal of Power Sources, 108 (2002) 153162.

[16] P.L. Taberna, P. Simon, J.F. Fauvarque Electrochemical Characteristics and Impedance Spectroscopy Studies of Carbon-Carbon Supercapacitors, Journal of The Electrochemical Society, 150 (2003) A292-A300.

[17] M. Ates, Review study of electrochemical impedance spectroscopy and equivalent electrical circuits of conducting polymers on carbon surfaces, Progress in Organic Coatings, 71 (2011) 1-10. [18] A. Lasia, Impedance of porous electrodes, Journal of Electroanalytical Chemistry, 397 (1995) 2733.

[19] B.E. Conway, Electrochemical supercapacitors: scientific fundamentals and technological applications, Springer Science \& Business Media2013. 
[20] S. Fletcher, I. Kirkpatrick, R. Dring, R. Puttock, R. Thring, S. Howroyd, The modelling of carbonbased supercapacitors: Distributions of time constants and Pascal Equivalent Circuits, Journal of Power Sources, 345 (2017) 247-253.

[21] S. Fletcher, Tables of Degenerate Electrical Networks for Use in the Equivalent-Circuit Analysis of Electrochemical Systems, Journal of The Electrochemical Society, 141 (1994) 1823-1826.

[22] S. Fletcher, V.J. Black, I. Kirkpatrick, A universal equivalent circuit for carbon-based supercapacitors, Journal of Solid State Electrochemistry, 18 (2014) 1377-1387.

[23] R. De Levie, Electrochemical response of porous and rough electrodes, Advances in electrochemistry and electrochemical engineering, 6 (1967) 329-397.

[24] H.-K. Song, Y.-H. Jung, K.-H. Lee, L.H. Dao, Electrochemical impedance spectroscopy of porous electrodes: the effect of pore size distribution, Electrochimica Acta, 44 (1999) 3513-3519.

[25] H.-K. Song, H.-Y. Hwang, K.-H. Lee, L.H. Dao, The effect of pore size distribution on the frequency dispersion of porous electrodes, Electrochimica Acta, 45 (2000) 2241-2257.

[26] A.M. Johnson, J. Newman, Desalting by Means of Porous Carbon Electrodes, Journal of The Electrochemical Society, 118 (1971) 510-517.

[27] J.W. Graydon, M. Panjehshahi, D.W. Kirk, Charge redistribution and ionic mobility in the micropores of supercapacitors, Journal of Power Sources, 245 (2014) 822-829.

[28] C. Portet, P.L. Taberna, P. Simon, C. Laberty-Robert, Modification of Al current collector surface by sol-gel deposit for carbon-carbon supercapacitor applications, Electrochimica Acta, 49 (2004) 905-912.

[29] H. Gualous, D. Bouquain, A. Berthon, J.M. Kauffmann, Experimental study of supercapacitor serial resistance and capacitance variations with temperature, Journal of Power Sources, 123 (2003) 86-93.

[30] X. Wang, D. Kong, Y. Zhang, B. Wang, X. Li, T. Qiu, Q. Song, J. Ning, Y. Song, L. Zhi, All-biomaterial supercapacitor derived from bacterial cellulose, Nanoscale, 8 (2016) 9146-9150.

[31] L. Sun, C. Tian, M. Li, X. Meng, L. Wang, R. Wang, J. Yin, H. Fu, From coconut shell to porous graphene-like nanosheets for high-power supercapacitors, Journal of Materials Chemistry $A, 1$ (2013) 6462-6470.

[32] Y. Lv, L. Gan, M. Liu, W. Xiong, Z. Xu, D. Zhu, D.S. Wright, A self-template synthesis of hierarchical porous carbon foams based on banana peel for supercapacitor electrodes, Journal of Power Sources, 209 (2012) 152-157.

[33] Q. Wang, Q. Cao, X. Wang, B. Jing, H. Kuang, L. Zhou, A high-capacity carbon prepared from renewable chicken feather biopolymer for supercapacitors, Journal of Power Sources, 225 (2013) 101-107.

[34] S. Fletcher, Contribution to the theory of conducting-polymer electrodes in electrolyte solutions, Journal of the Chemical Society, Faraday Transactions, 89 (1993) 311-320.

[35] F.A. Posey, T. Morozumi, Theory of Potentiostatic and Galvanostatic Charging of the Double Layer in Porous Electrodes, Journal of The Electrochemical Society, 113 (1966) 176-184.

[36] D.D. Macdonald, Reflections on the history of electrochemical impedance spectroscopy, Electrochimica Acta, 51 (2006) 1376-1388.

[37] D.C. Grahame, The Electrical Double Layer and the Theory of Electrocapillarity, Chemical Reviews, 41 (1947) 441-501.

[38] R. De Levie, On porous electrodes in electrolyte solutions -IV, Electrochimica acta, 9 (1964) 1231-1245.

[39] O.E. Barcia, E. D'Elia, I. Frateur, O.R. Mattos, N. Pébère, B. Tribollet, Application of the impedance model of de Levie for the characterization of porous electrodes, Electrochimica acta, 47 (2002) 2109-2116.

[40] M. Zhi, C. Xiang, J. Li, M. Li, N. Wu, Nanostructured carbon-metal oxide composite electrodes for supercapacitors: a review, Nanoscale, 5 (2013) 72-88. 
[41] X. Gao, W. Xing, J. Zhou, G. Wang, S. Zhuo, Z. Liu, Q. Xue, Z. Yan, Superior capacitive performance of active carbons derived from Enteromorpha prolifera, Electrochimica Acta, 133 (2014) 459-466.

[42] B.G. Choi, J. Hong, W.H. Hong, P.T. Hammond, H. Park, Facilitated Ion Transport in All-SolidState Flexible Supercapacitors, ACS Nano, 5 (2011) 7205-7213.

[43] P. Zhang, F. Sun, Z. Shen, D. Cao, ZIF-derived porous carbon: a promising supercapacitor electrode material, Journal of Materials Chemistry A, 2 (2014) 12873-12880.

[44] J.S. Lee, D.H. Shin, J. Jang, Polypyrrole-coated manganese dioxide with multiscale architectures for ultrahigh capacity energy storage, Energy \& Environmental Science, 8 (2015) 3030-3039.

[45] V.K. Tiwari, Z. Chen, F. Gao, Z. Gu, X. Sun, Z. Ye, Synthesis of ultra-small carbon nanospheres $(<50 \mathrm{~nm})$ with uniform tunable sizes by a convenient catalytic emulsion polymerization strategy: superior supercapacitive and sorption performance, Journal of Materials Chemistry A, 5 (2017) 12131-12143.

[46] M. Alhabeb, M. Beidaghi, K.L. Van Aken, B. Dyatkin, Y. Gogotsi, High-density freestanding graphene/carbide-derived carbon film electrodes for electrochemical capacitors, Carbon, 118 (2017) 642-649.

[47] W. Yang, W. Yang, F. Ding, L. Sang, Z. Ma, G. Shao, Template-free synthesis of ultrathin porous carbon shell with excellent conductivity for high-rate supercapacitors, Carbon, 111 (2017) 419-427. [48] M. Barczak, Y. Elsayed, J. Jagiello, T.J. Bandosz, Exploring the effect of ultramicropore distribution on gravimetric capacitance of nanoporous carbons, Electrochimica Acta, 275 (2018) 236247.

[49] J.W. Lee, A.S. Hall, J.-D. Kim, T.E. Mallouk, A Facile and Template-Free Hydrothermal Synthesis of Mn3O4 Nanorods on Graphene Sheets for Supercapacitor Electrodes with Long Cycle Stability, Chemistry of Materials, 24 (2012) 1158-1164.

[50] A. Ramadoss, K.-Y. Yoon, M.-J. Kwak, S.-I. Kim, S.-T. Ryu, J.-H. Jang, Fully flexible, lightweight, high performance all-solid-state supercapacitor based on 3-Dimensional-graphene/graphite-paper, Journal of Power Sources, 337 (2017) 159-165.

[51] D.I. Abouelamaiem, L. Rasha, G. He, T.P. Neville, J. Millichamp, T.J. Mason, A.B. Jorge, I.P. Parkin, M.-M. Titirici, R. Wang, S. Ji, P.R. Shearing, D.J.L. Brett, Integration of supercapacitors into printed circuit boards, Journal of Energy Storage, 19 (2018) 28-34. 